UNITED STATES

DEPARTMENT OF INTERIOR

GEOLOGICAL SURVEY

TIME VARYING SPECTRAL CHARACTERISTICS OF STRONG GROUND MOTIONS: THE IMPERIAL VALLEY, CALIFORNIA EARTHQUAKE OF OCTOBER 15, 1979

\author{
Erdal Şafak \\ Research Structural Engineer \\ U. S. Geological Survey, Menlo Park, California 94025 \\ Fouad M. Bendimerad \\ Post Doctorate Research Affiliate \\ Department of Civil Engineering, Stanford University, Stanford, California 94305
}

Open-File Report 86-443

This report is preliminary and has not been reviewed for conformity with U.S. Geological Survey editorial standards and stratigraphic nomenclature

August 27, 1986 


\section{TIME VARYING SPECTRAL CHARACTERISTICS OF STRONG GROUND MOTIONS: THE IMPERIAL VALLEY, CALIFORNIA EARTHQUAKE OF OCTOBER 15, 1979}

\section{INTRODUCTION}

The instruments used for strong ground motion measurements record time variation of ground acceleration, velocity, or displacement. The frequency content of the motion is determined from the Fourier spectrum obtained by taking the Fourier transform of the recorded time series. The Fourier spectrum is a complex quantity that consists of two parts, the amplitude spectrum, and the phase spectrum. The amplitude spectrum gives a measure of contribution from each frequency to the total energy (i.e. mean square value) of the signal, whereas the phase spectrum gives a measure of the transient properties of the signal. There is a one-to-one correspondance between the time series and its complex Fourier spectrum. In other words, any given pair of amplitude and phase spectra corresponds to a specific time series, and any given time series correspond to a specific pair of amplitude and phase spectra. Most of the past research has been concentrated on the amplitude spectrum. There are analytical models developed that can approximate the amplitude spectrum in terms of source, path, and site parameters (see Boore, 1986 for an extensive review and references). Without the phase spectrum, however, the amplitude spectrum alone does not provide any information on the transient characteristics of the motion. Studies on recorded ground motions indicate that the phase spectrum is very random, especially for large magnitude earthquakes; thus, it is hard to parameterize or to develope an approximate analytical model.

An alternate way of tracking transient characteristics of ground motions is to take the Running Fourier Transform, instead of the ordinary Fourier transform of the recorded time series. The running Fourier transform is the Fourier transform taken after multiplication of the original time series by a running window. Thus, the running Fourier transform is not only a function of frequency, but also function of time, defined by the location and the shape of the window. The running Fourier transform leads to the development of the Physical Spectra, or the Time-Varying Spectra, which is the extension of the concept of power spectra for stationary time series to nonstationary time series. The physical spectra give the distribution of energy with time and frequency, and allow to track the variation of frequency characteristics of the motion with time, as well as the variation of temporal characteristics with frequency. The word energy used here refers to the energy of the time series (i.e. its mean square value), not to the energy of the ground motion, unless the time series represent the ground velocity.

In this report, first the theoretical basis and derivations for the concepts of the run- 
ning Fourier transform and time-varying spectra are presented. Then, its application for studying two-component horizontal ground motions and their correlations is outlined. An example for the concept is given by using a data set from the October 15, 1979 Imperial Valley, California earthquake. The results from this methodology can have various applications. In particular, they can be used to calculate structural response under multicomponent ground excitation by using random vibration theory. To give an example, equations for the response of a 2-DOF oscillator under arbitrarily oriented bi-directional base excitation are given in Appendix-I at the end of the report.

\section{TIME-VARYING POWER SPECTRUM}

One of the approaches to study nonstationary time series is to calculate the timevarying spectrum (i.e. physical spectrum) of the time series. In this section, a short summary of the concept of time-varying spectrum will be given. A more detailed treatment of the subject can be found in a long paper by Mark (1970).

Consider a process $\left\{x(t), t=\left[0, T_{d}\right]\right\}$ representing an ensemble of acceleration time histories of duration $T_{d}$, and a window function $w(t), t=\left[-T_{w}, T_{w}\right]$. Define a new function $r(s, t)$ by taking the product

$$
r(s, t)=x(s) w(t-s)
$$

$r(s, t)$ corresponds to the acceleration time history isolated by the window function in the neighborhood of time $t$. The corresponding spectral content of $r(s, t)$ is obtained through the Fourier transform

$$
F(f, t ; w)=\int_{0}^{\infty} r(t, s) \exp (-i 2 \pi f s) d s=\int_{0}^{\infty}[x(s) w(t-s)] \exp (-i 2 \pi f s) d s
$$

In Eq. $2 f$ represents the frequency and $i=\sqrt{-1}$. Eq. 2 is referred to as the Running Fourier Transform because of its dependence on the time $t$. For a discrete time series one can generate as many Fourier transforms as there are number of points in the time series. Note that the index $w$ is left in the argument of $F$ to show that $F$ depends on the characteristics of $w$.

The concept of power spectrum for stationary processes can be extended to nonstationary processes by making use of the running Fourier transform, provided that $w(t)$ is properly defined. Let $U(t)$ denote the instantaneous power of $x(t)$ at time $t . U(t)$ is defined by the equation 


$$
U(t)=\int_{-\infty}^{+\infty} \mathbf{E}\left[F(f, t ; w) F^{*}(f, t ; w)\right] d f
$$

where superscript $*$ denotes the conjugate and $\mathbf{E}$ denotes the ensemble average. The integrand in Eq. 3 has the same formal definition as the power spectrum. It represents the frequency distribution of the energy of $x(t)$ in the neighborhood of $t$ as defined by $w(t)$. This integrand is referred to as a physical spectrum and is formally defined by the expression

$$
\begin{aligned}
S_{x}(f, t ; w) & =\mathbf{E}\left[F(f, t ; w) F^{*}(f, t ; w)\right] \\
& =\mathbf{E}\left\{\left|\int_{0}^{\infty}[x(s) w(t-s)] \exp (-i 2 \pi f s) d s\right|^{2}\right\}
\end{aligned}
$$

$S_{x}$ is a real positive quantity and an even function of $f$ for all values of $t$ and for any choice of $w$. The total energy $V$ (i.e. the energy of the time series, not necessarely the energy of the ground motion) of the process $\{x(t)\}$ is defined by the expression

$$
V=\int_{0}^{\infty} U(t) d t=\int_{-\infty}^{+\infty} \int_{-\infty}^{+\infty} S_{x}(f, t ; w) d f d t
$$

Hence, $S_{x}(f, t ; w)$ represents a simultaneous decomposition of the expected energy with time and frequency. If $t$ and $f$ are assumed to be the horizontal coordinates, and $S_{x}$ the vertical coordinate, then the volume under $S_{x}$ gives the total energy of the signal. It can be shown by using the Parseval's theorem that

$$
\int_{0}^{\infty} x^{2}(t) d t=\int_{-\infty}^{+\infty} \int_{-\infty}^{+\infty} S_{x}(f, t ; w) d f d t=V
$$

which gives the normalization condition for $w(t)$. By using Eq. 1 in Eq. 6, the normalization condition is obtained as

$$
\int_{-\infty}^{+\infty}[w(t)]^{2} d t=1
$$

The preceeding development defining the physical spectrum and related concepts would have equally been established from a frequency domain approach. If $W(f)$ is the Fourier transform of $w(t)$, then by virtue of Parseval's theorem and Eq. 7 


$$
\int_{-\infty}^{+\infty}|W(f)|^{2} d f=1
$$

It can be proven by using the frequency convolution theorem that the frequency-based definition of the physical spectrum is given by

$$
S_{x}(f, t ; w)=E\left\{\left|\int_{-\infty}^{+\infty} W(f-g) X^{*}(g) \exp (-i 2 \pi g t) d g\right|^{2}\right\}
$$

where $X(g)$ is the Fourier transform of $x(t)$. The expected energy spectrum in the neighborhood of the frequency $f$ is given by

$$
Q(f)=\int_{-\infty}^{+\infty} S_{x}(f, t ; w) d t
$$

This indicates that $S_{x}$ can also be interpreted as a non-negative time decomposition of the locally averaged value of the expected energy spectrum in the neighborhood of $f$. The concept of physical spectra has been used in stochastic modeling of earthquake ground motions (Tilliouine, 1982; Bendimerad, 1984), and in wave studies of earthquake time histories (Hoshiya, 1979).

The concept of time-varying spectrum described above for a single time series (i.e. one-directional ground motion) can easily be extended to two-directional time series. If $x(t)$ and $y(t)$ denote the orthogonal components of the horizontal ground motion, the timevarying spectral resolution of the motion can be defined by a two-dimensional matrix [S]. The diagonal elements $S_{x}$ and $S_{y}$ of [S] denote the physical spectra of $x(t)$ and $y(t)$ as defined by Eq. 4 or Eq. 9 ; the off-diagonal elements $S_{x y}$ and $S_{y x}$ of [S] denote the cross (i.e. non-physical) spectra. $S_{x y}$ is given by

$$
\begin{aligned}
S_{x y}(f, t ; w)=E\{ & {\left[\int_{-\infty}^{+\infty} x(s) w(t-s) \exp (-i 2 \pi f s) d s\right] \times } \\
& {\left.\left[\int_{-\infty}^{+\infty} y(s) w(t-s) \exp (-i 2 \pi f s) d s\right]\right\} . }
\end{aligned}
$$


As explained earlier the physical spectra $S_{x}$ and $S_{y}$ are always positive. The non-physical spectra $S_{x y}$ and $S_{y x}$ are generally complex quantities. However, one can easily show that

$$
S_{x y}(f, t ; w)=S_{y x}^{*}(f, t ; w)
$$

so that

$$
\left|S_{x y}(f, t ; w)\right|=\left|S_{y x}(f, t ; w)\right|
$$

That is, $S_{x y}$ and $S_{y x}$ have the same amplitude spectrum. Hence, if the phase of $S_{x y}$ and $S_{y x}$ are not of concern, only three spectra (two physical spectra and one cross spectrum) are necessary to define the time-dependent resolution of the two-directional horizontal ground motions.

The preceding developments pertaining to the physical spectrum and related concepts have been presented from a stochastic approach. It must be clear, however, that when the ensemble average symbol $\mathbf{E}$ is removed from the equations, all the definitions and their properties are still valid for a single time series $x(t)$.

\section{SELECTION AND CHARACTERISTICS OF THE WINDOW FUNCTION}

In the preceding sections, it was pointed out that the physical spectrum depends on the choice of the window function. This can be explained from the interpretation of Eqs. 4 and 9. In Eq. $4, S_{x}(f, t ; w)$ can be interpreted as the description of the properties of $x(t)$ at time $t$ in a small time window prescribed by $w(t)$, whereas in Eq. 9 , it is the description of the properties of $X(f)$ at frequency $f$ about a small frequency window prescribed by $W(f)$. Thus, the extent and character of this time and frequency smearing is controlled by the window function pair $w(t)$ and $W(f)$.

Basically, windowing forces us to look at the data through a narrow strip. Intuitively, we would like to choose the window function so as to capture the detailed properties of the signal in the time domain, and at the same time, keep the bandwidth of the window spectrum narrow enough to insure high frequency resolution. As it is well known, this can not be achieved in both domains simultaneously, because of the inherent property of the Fourier decomposition that sharp phenomena in the time domain results in broad spectra in the frequency domain, and vice-versa. Phrased differently, the larger the length of the window $w(t)$, the narrower the frequency width of $W(f)$; and consequently, the more accurate spectral decompositon, but less accurate time resolution.

Thus, in generating a particular $S(f, t ; w)$ it is possible to specify any desired fine time resolution, at the expense of the frequency resolution; or, any desired frequency resolution, at the expense of the time resolution. The question is then how to choose a Fourier window pair that provides an optimum simultaneous time and frequency resolution. Unfortunately, 
there is no clear-cut answer to this question. The answer is generally different for each specific signal, and is usually dictated by the nature of the problem.

Although there is no specific theory of window selection, one should look for trends and establish guidelines in order to arrive at an acceptable choice for the window function. For example, it is clear that a pulse or a rectangular function is a poor choice for a window, because of sharp discontinuities at the ends. We have tested various types of window functions for processing earthquake accelerograms, and investigated their time and frequency resolutions. Following are the guidelines that we propose:

1. The window should be a smooth function that highly concentrates on the point about which the average is taken, but yet has a width large enough to insure an acceptable spectral resolution. The justification for this point should be clear from the preceding discussion.

2. The window should have tails that gradually reduces the signal to zero (or to a value very close to zero) at the truncation points. Windowing introduces a sharp discontinuity at each end-point of the window. The Fourier transform is very sensitive to such discontinuity which causes Gibbs effects or ringing. A window with rounded corners that gradually taper at the ends reduces that effect.

3. It is advantageous to choose a real and even window function, so that the Fourier transform would also be real and even. This can save a considerable amount of computation time.

4. Finally, we must recall that the window function must satisfy the normalization condition given by Eqs. 7 and 8.

A class of functions that fit the proposed guidelines quite well is that of the Gaussian type. These functions have the property of being self-reciprocal; that is, the Fourier transform of a Gaussian function is also Gaussian. We select the following pair of Gaussian type functions to represent windows in time and frequency domains

$$
\begin{gathered}
w(t)=\frac{1}{(\pi)^{1 / 4} \sqrt{\sigma_{t}}} \exp \left(-\frac{t^{2}}{2 \sigma_{t}^{2}}\right) \\
W(f)=\frac{1}{(\pi)^{1 / 4} \sqrt{\sigma_{f}}} \exp \left(-\frac{f^{2}}{2 \sigma_{f}^{2}}\right)
\end{gathered}
$$

$\sigma_{t}$ and $\sigma_{f}$ denote the standard deviations (i.e. half of the distance between the inflection points) of Gaussian type window functions in time and frequency domains, respectively. They will be referred as the half-widths of the windows. It can be shown by straight 
forward integration that $w(t)$ and $W(f)$ are Fourier transform pairs, and

$$
\sigma_{t} \cdot \sigma_{f}=\frac{1}{2 \pi}
$$

Eq. 16 clearly shows why an increase in the resolution in one domain causes a decrease in the resolution in the other domain (i.e. a decrease in the time width causes an increase in the frequency width and vice-versa). The values of window functions at the origin are

$$
w(0)=\frac{1}{\pi^{1 / 4} \sqrt{\sigma_{t}}} \quad \text { and } \quad W(0)=\frac{1}{\pi^{1 / 4} \sqrt{\sigma_{f}}}
$$

Eqs. 14 and 15 satisfy the normalization conditions

$$
\begin{aligned}
& \int_{-\infty}^{+\infty} w^{2}(t) d t=\int_{-\infty}^{+\infty} \frac{1}{\sqrt{\pi} \sigma_{t}} \exp \left(-\frac{t^{2}}{\sigma_{t}^{2}}\right) d t=1 \\
& \int_{-\infty}^{+\infty} W^{2}(f) d f=\int_{-\infty}^{+\infty} \frac{1}{\sqrt{\pi} \sigma_{f}} \exp \left(-\frac{f^{2}}{\sigma_{f}^{2}}\right) d f=1
\end{aligned}
$$

A simple way of proving Eqs. 18 and 19 is to note that the integrands are Gaussian distribution functions with means equal to zero and standard deviations equal to $\sigma_{t} / \sqrt{2}$ and $\sigma_{f} / \sqrt{2}$, respectively. Thus, their integral from $-\infty$ to $+\infty$ are equal to one.

The window function needs to be truncated such that it gradually reduces the signal to zero at the window end-points. The Gaussian function, however, approaches to zero only in the limit case (i.e. as $t \rightarrow \infty$ ). For simplicity, we represent the length of the window as a multiple of its width. We also require that the number of discrete points within the window is equal to a power of two, so that the FFT (Fast Fourier Transform) techniques can be used. Mathematically, these conditions are given in the time domain as

$$
L_{t}=2^{p} \cdot \Delta t \geq k \cdot 2 \sigma_{t}
$$

where $L_{t}$ is the window length, $\Delta t$ is the sampling interval, and $p$ and $k$ are integers. For a specified value of $L_{t}$, the frequency resolution of the window (i.e. the lower and upper bounds of the frequency band that can be resolved) is described by

$$
f_{\min }=\frac{1}{L_{t}} \quad \text { and } \quad f_{\max }=\frac{1}{2 \Delta t}
$$

Thus, in a time series of given $\Delta t$, by specifying only the window length $L_{t}$ (or the window half-width $\sigma_{t}$ and integer $k$ ) all the characteristics of the time-frequency resolution of the signal are defined. 


\section{APPLICATION TO IMPERIAL VALLEY EARTHQUAKE DATA}

The concept of physical spectrum described above is applied to a data set from October 15, 1979, Imperial Valley, California earthquake. The data set consists of three-component acceleration records from 22 stations. Only horizontal components of the recordings are considered. In each station, the time-varying spectrum for horizontal components and their cross spectrum are calculated by using the methodology described above.

First, the original data, which had a sampling rate of 100 points-per-second (corresponding frequency band is $50 \mathrm{~Hz}$ ), were filtered with a bi-directional Butterworth filter and decimated to 25 samples-per-second (corresponding frequency band is $12.5 \mathrm{~Hz}$ ). This is done to reduce the computational work, and is justifed since the frequency band that is of interest in engineering design is usually less than $10 \mathrm{~Hz}$. After testing several alternatives, the window half-width is taken as $\sigma_{t}=1.06$ seconds, and the window length is assumed to be five times of the window width (i.e. $10 \sigma_{t}$ ), which gives $L_{t}=10.6$ seconds, and is equivalent to 256 discrete time points. From Eq. 2, by putting $t=5 \sigma_{t}$ it can be shown that above values correspond to an amplitude reduction of $3.7 \times 10^{-6}$ (i.e. $e^{-12.5}$ ) of the peak at the truncation points of the window. The frequency resolution, from Eq. 21 , is $f_{\min }=0.094 \mathrm{~Hz}$ and $f_{\max }=12.5 \mathrm{~Hz}$.

The results are plotted in Figs. 2 for all the stations, which include recorded horizontal acceleration time histories, time-varying spectra of each component, and the cross spectra for each station. The figures clearly show that the spectral characteristics of the motion change with time. It is also clear from the figures that the horizontal components are highly correlated at certain frequency and time bands.

The time-varying spectra and cross spectra present significant information on the rupture process of the earthquake and its propagation. The extraction of this information requires a close inspection of all the plots, incorporating the information on the epicentral distances, site conditions and recording directions. This work is currently in progress.

Another use of the two-dimensional time varying spectrum is the calculation of bidirectional response of structures under multi-component base excitation. This problem has been investigated by using a statistical approach by Safak and Bendimerad (1986). The problem can also be addressed analytically by using the random vibration theory. Equations for the response of a 2-DOF oscillator under arbitrarily oriented bi-directional base motion are given in Appendix-I at the end of the report.

\section{SUMMARY AND CONCLUSIONS}

The concepts of running Fourier transform and time-varying spectrum, and their use in analyzing ground motion recordings are presented. The running Fourier transform is the Fourier transform taken after multiplication of the original time series by a running time window. By using the running Fourier transforms, time-varying spectral characteristics 
of ground motions can be investigated. The window function should satisfy certain conditions, so that optimum resolutions in frequency and time domains are obtained. These conditions are presented in the report. The concept is applied to a data set from the October 15, 1979 Imperial Valley, California earthquake. The time-varying spectra and cross-spectrum for horizontal components of the recorded motions at 22 stations are calculated, and plotted in Figs. 2. The results show that the spectral content of the motion varies with time. The results also show that the correlation of the horizontal components are both time and frequency dependent. The methodology can have various practical applications. In particular, it can be used to calculate structural response under multicomponent ground excitation. To give an example, the response of a 2-DOF oscillator subjected to bi-directional, arbitrarily oriented base excitation is presented in Appendix-I.

\section{ACKNOWLEDGEMENT}

The authors would like to thank to W.B. Joyner of the U.S. Geological Survey, Menlo Park, for providing the data set from the Imperial Valley, California earthquake. 


\section{REFERENCES}

1. Bendimerad, M.F. (1984). Modeling of recorded 3-dimensional earthquake motion in the frequency-time domain, Ph.D. thesis, Civil Engineering Department, Stanford University, Stanford, California.

2. Boore, D.M. (1986). The prediction of strong ground motion, proceedings of NATO Advanced Studies Institute on Strong Ground Motion Seismology, June 10-21, 1985, Ankara, Turkey.

3. Hoshiya, M. (1979). Principal axes and wave characteristics of earthquake ground motion, proc. of the 3rd International Conference on the Applications of Statistics and Probability to Soils and Structural Engineering, Sydney, Australia, Vol.2, pp.765771.

4. Mark, W.D. (1970). Spectral analysis of the convolution and filtering of nonstationary stochastic processes, Journal of Sound and Vibrations, Vol.11, No.1, pp.19-63.

5. Tiliouine, B. (1982): Nonstationary analysis and simulation of seismic signals, Ph.D. thesis, Department of Civil Engineering, Stanford University, Stanford, California.

6. Safak, E. and M.F. Bendimerad (1986). Peak response of 2-DOF oscillator under twodirectional base motion, submitted for publication to ASCE Journal of Engineering Mechanics. 


\section{APPENDIX-I. RESPONSE OF 2-DOF OSCILLATOR TO ARBITRARILY ORIENTED TWO-DIRECTIONAL BASE MOTION}

Figure A.1 shows the schematic of the problem, and the notation. The base accelerations in $x$ and $y$ directions are

$$
\begin{aligned}
& a_{x}=-a_{1} \cos \theta+a_{2} \sin \theta \\
& a_{y}=-a_{1} \sin \theta-a_{2} \cos \theta
\end{aligned}
$$

Assuming that there is no torsional vibrations, the equations for the vibration of the oscillator in $x$ and $y$ directions are

$$
\begin{aligned}
& \ddot{x}+2 \xi_{x}\left(2 \pi f_{x}\right) \dot{x}+\left(2 \pi f_{x}\right)^{2} x=a_{x} \\
& \ddot{y}+2 \xi_{y}\left(2 \pi f_{y}\right) \dot{y}+\left(2 \pi f_{y}\right)^{2} y=a_{y}
\end{aligned}
$$

where $f_{x}, f_{y}$ are the natural frequencies, and $\xi_{x}, \xi_{y}$ are the damping ratios for $x$ and $y$ directions. By using the methods of random vibration theory, the time-varying spectra of the response for the $x$ direction, $S_{x x}(f, t)$ can be calculated from the following equation

$$
S_{x x}(f, t)=\int_{-\infty}^{+\infty} S_{a_{x} a_{x}}(f, t) M_{x}(f, t-u) d u
$$

where $S_{a_{x} a_{x}}(f, t)$ denotes the time-varying spectra of acceleration component $a_{x} . M_{x}(f, t)$ is defined as

$$
M_{x}(f, t)=\int_{-\infty}^{+\infty} H_{x}\left(f-\frac{g}{2}\right) H_{x}^{*}\left(f+\frac{g}{2}\right) \exp (-i 2 \pi g t) d g
$$

$H_{x}(f)$ is the frequency response function for the $x$ direction, and is given by

$$
H_{x}(f)=\left[-(2 \pi f)^{2}+i 2 \xi_{x}\left(2 \pi f_{x}\right)(2 \pi f)+\left(2 \pi f_{x}\right)^{2}\right]^{-1}
$$

Similarly, for the response in the $y$ direction

$$
S_{y y}(f, t)=\int_{-\infty}^{+\infty} S_{a_{y} a_{y}}(f, t) M_{y}(f, t-u) d u
$$

where

$$
M_{\nu}(f, t)=\int_{-\infty}^{+\infty} H_{y}\left(f-\frac{g}{2}\right) H_{y}^{*}\left(f+\frac{g}{2}\right) \exp (-i 2 \pi g t) d g
$$


and

$$
H_{y}(f)=\left[-(2 \pi f)^{2}+i 2 \xi_{y}\left(2 \pi f_{y}\right)(2 \pi f)+\left(2 \pi f_{y}\right)^{2}\right]^{-1}
$$

In order to complete the calculations, $S_{a_{x} a_{x}}$ and $S_{a_{y} a_{y}}$ need to be calculated in terms of the time-varying spectra and cross spectra of the excitation $a_{1}$ and $a_{2}$. It can be written from Eqs. A.1 and A.2 that

$$
\begin{aligned}
& S_{a_{x} a_{x}}(f, t)=S_{a_{1} a_{1}}(f, t) \cos ^{2} \theta+S_{a_{2} a_{2}}(f, t) \sin ^{2} \theta+\left[S_{a_{1} a_{2}}(f, t)+S_{a_{2} a_{1}}(f, t)\right] \sin \theta \cos \theta \\
& S_{a_{y} a_{y}}(f, t)=S_{a_{1} a_{1}}(f, t) \sin ^{2} \theta+S_{a_{2} a_{2}}(f, t) \cos ^{2} \theta+\left[S_{a_{1} a_{2}}(f, t)+S_{a_{2} a_{1}}(f, t)\right] \sin \theta \cos \theta
\end{aligned}
$$

$S_{a_{1} a_{1}} S_{a_{2} a_{2}}$ and $S_{a_{1} a_{2}}$ are calculated in terms of running Fourier transforms of $a_{1}$ and $a_{2}$ as given by Eqs. 4 and 11. $S_{x x}$ and $S_{y y}$ give all the information on the second order statistics of the response. 


\section{FIGURE CAPTIONS}

FIG. 1. Gaussian-type window function and its Fourier amplitude spectrum.

FIG. 2. Horizontal acceleration records, and their time-varying power spectra and cross spectrum from The Imperial Valley, California, Earthquake of October 15, 1979 (22 stations).

FIG. A.1. Schematic of a 2-DOF oscillator subjected to arbitrarily oriented bi-directional base motion. 

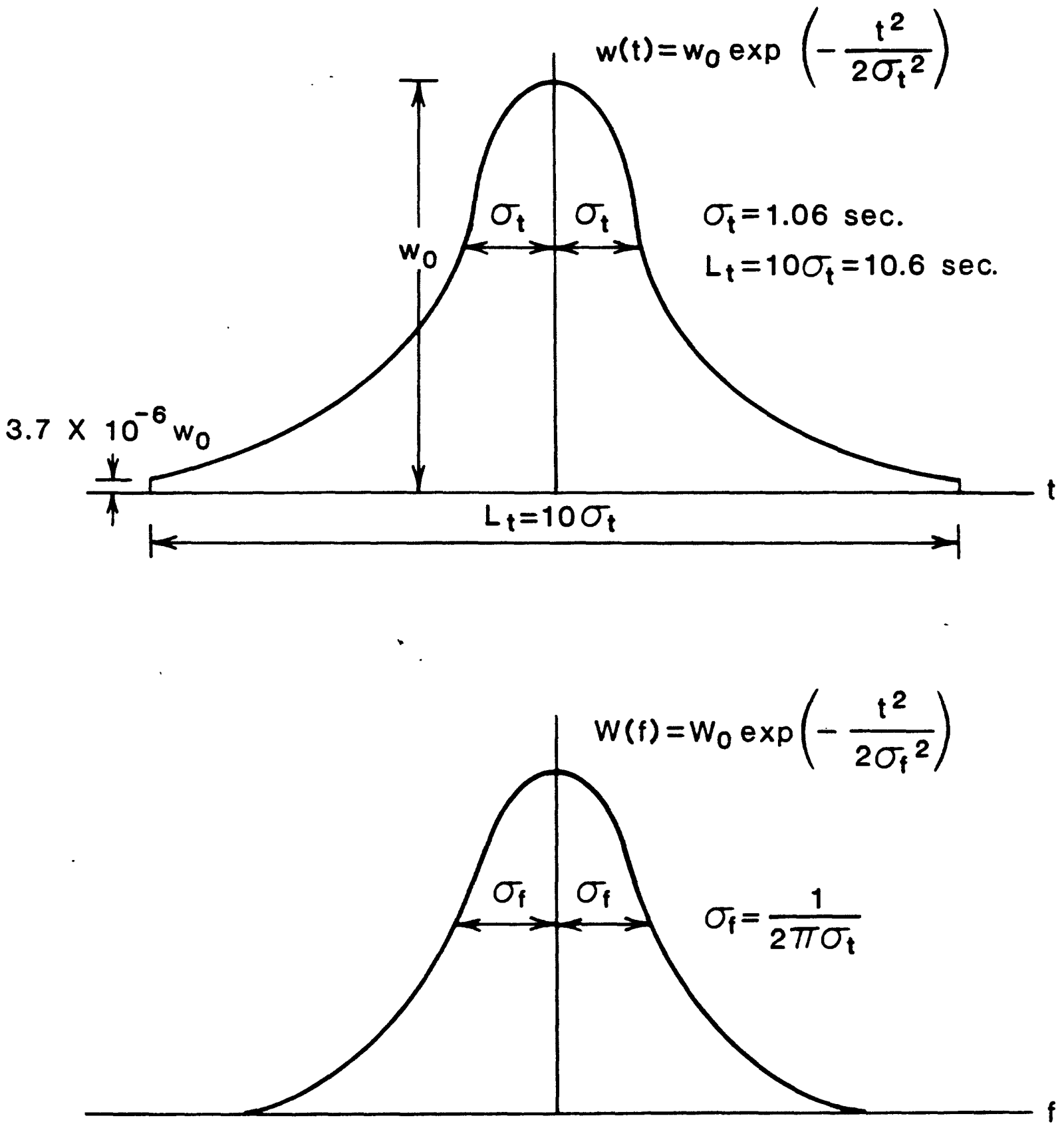

FIG. 1. Gaussian-type window function and its Fourier amplitude spectrum 

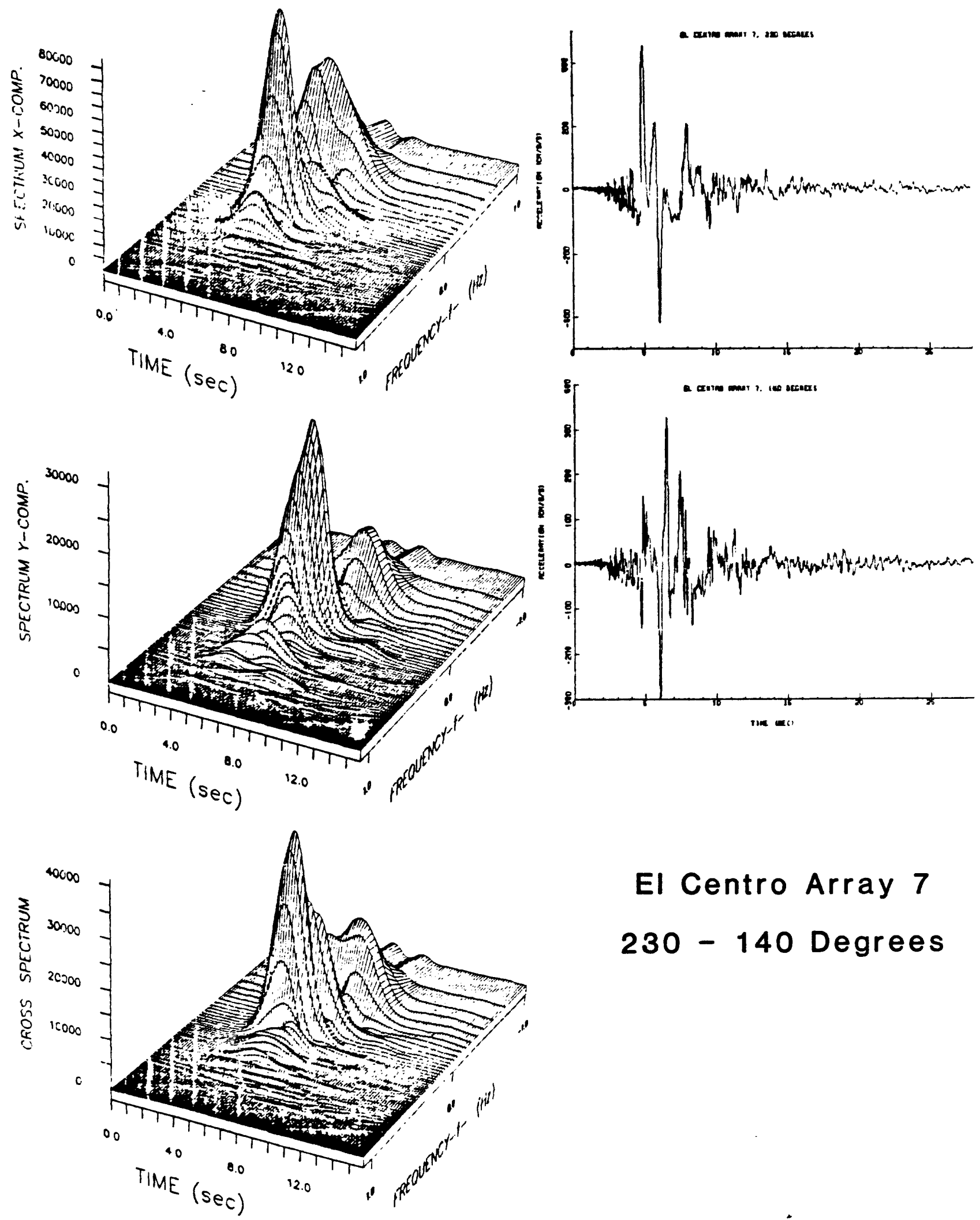

\section{El Centro Array 7 230 - 140 Degrees}

FIG. 2. Horizontal acceleration records and their time-varying power spectra and cross-spectra cross-spectrum from the Imperial Valley, California earthquake of October 15,1979 (22 stations). 

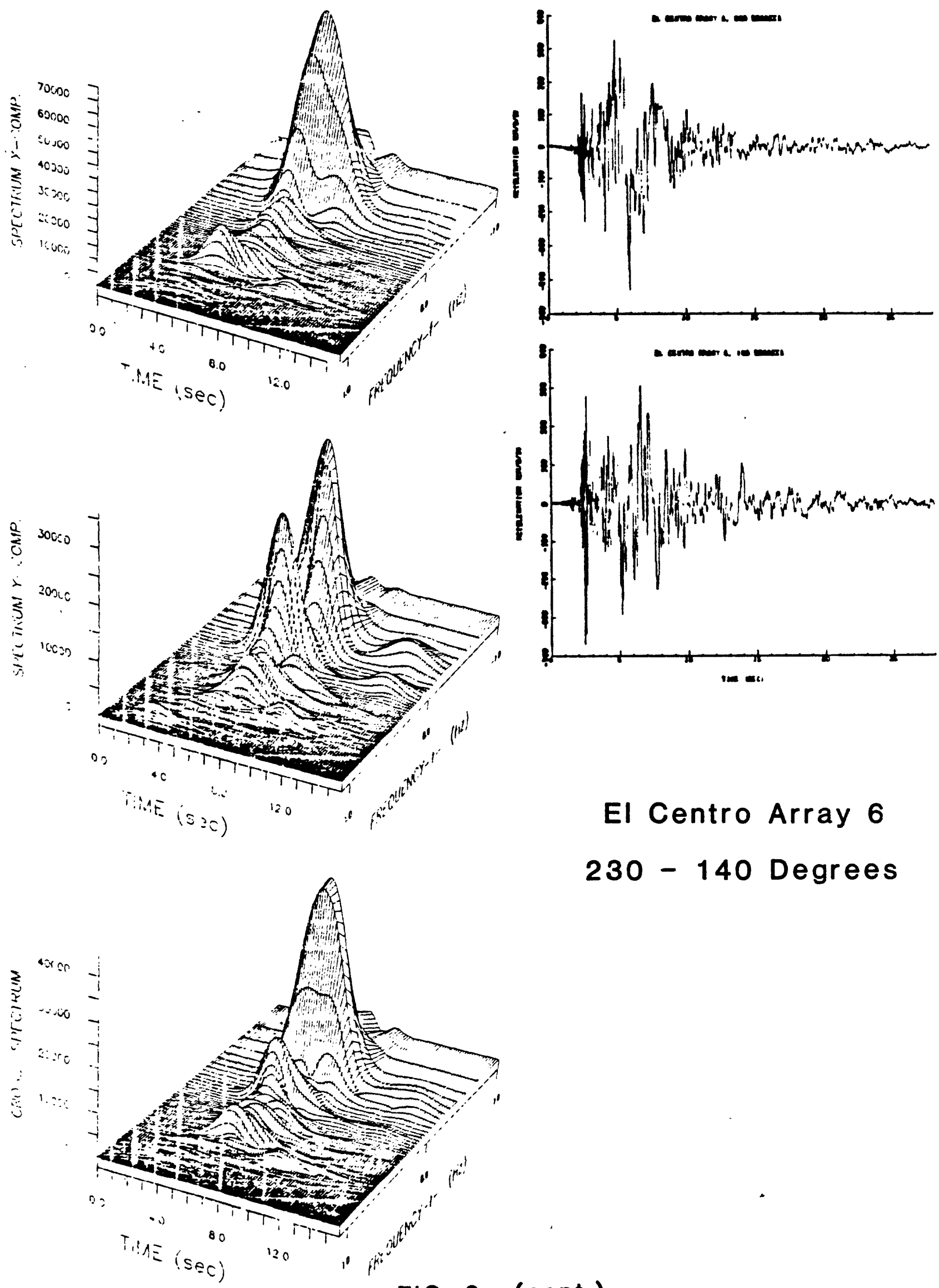

\section{El Centro Array 6 230 - 140 Degrees}

\footnotetext{
FIG. 2. (cont.)
} 

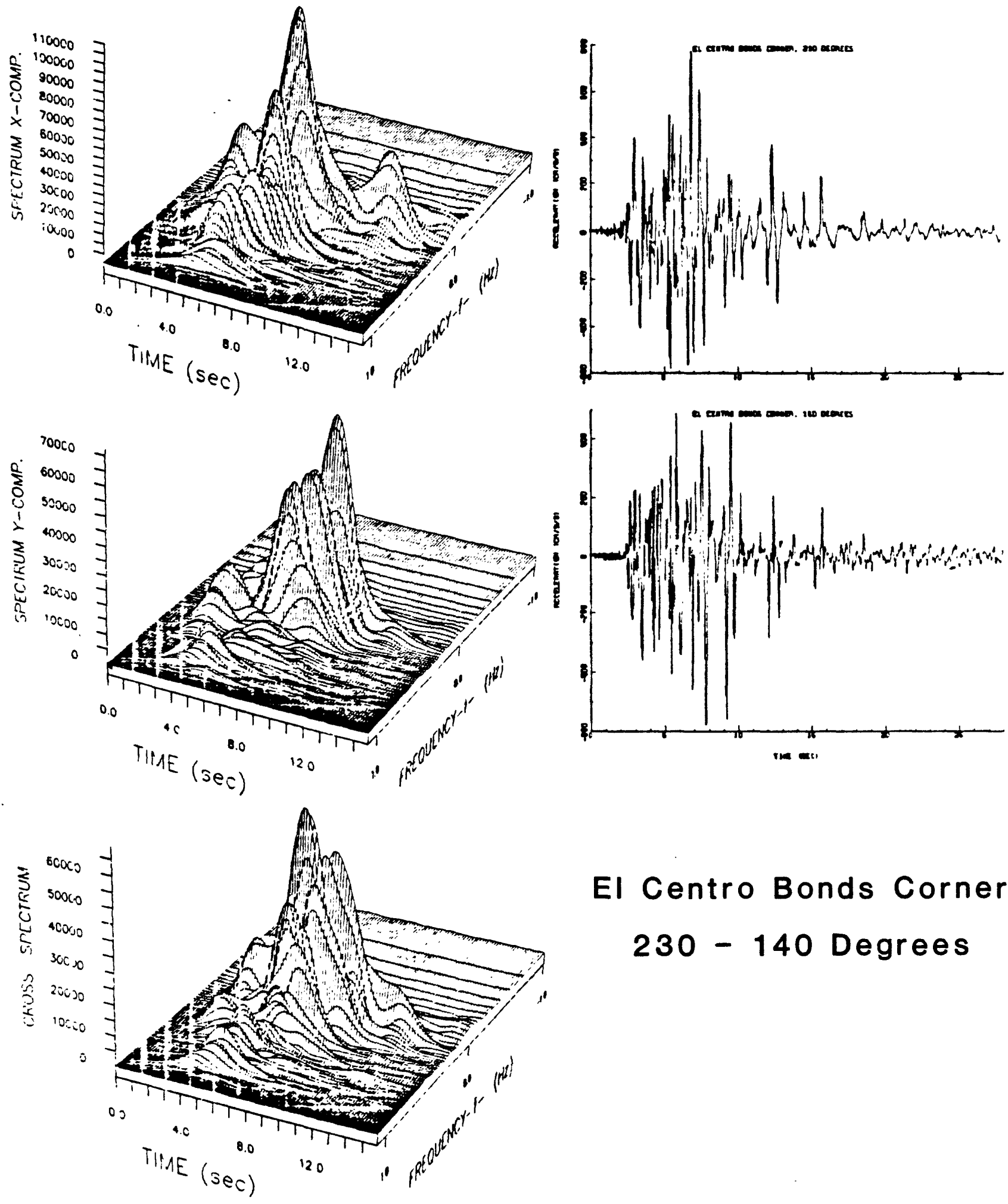

\section{El Centro Bonds Corner 230 - 140 Degrees}

FIG. 2. (cont.) 

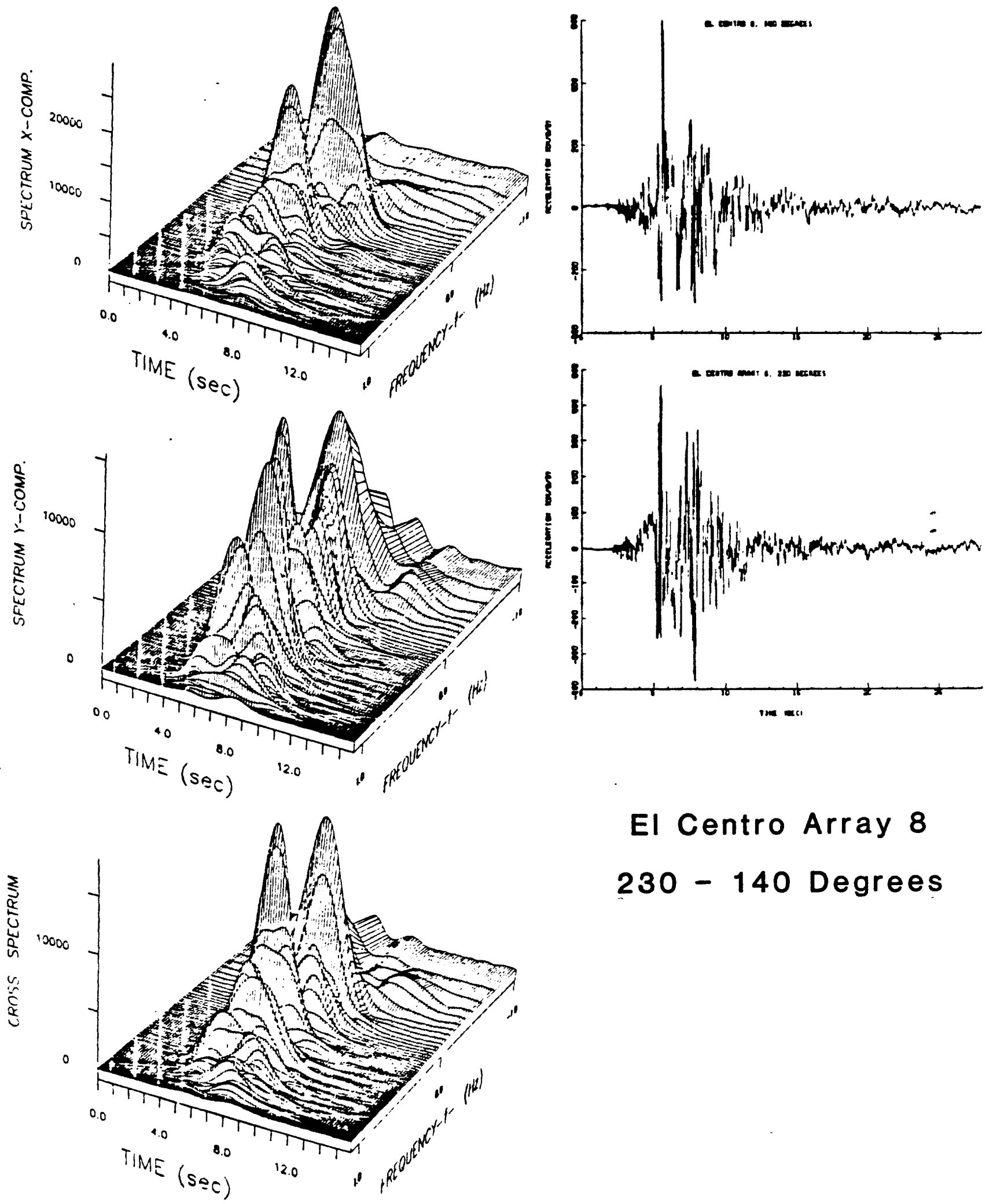

\section{El Centro Array 8 \\ 230 - 140 Degrees}

FIG. 2. (cont.) 

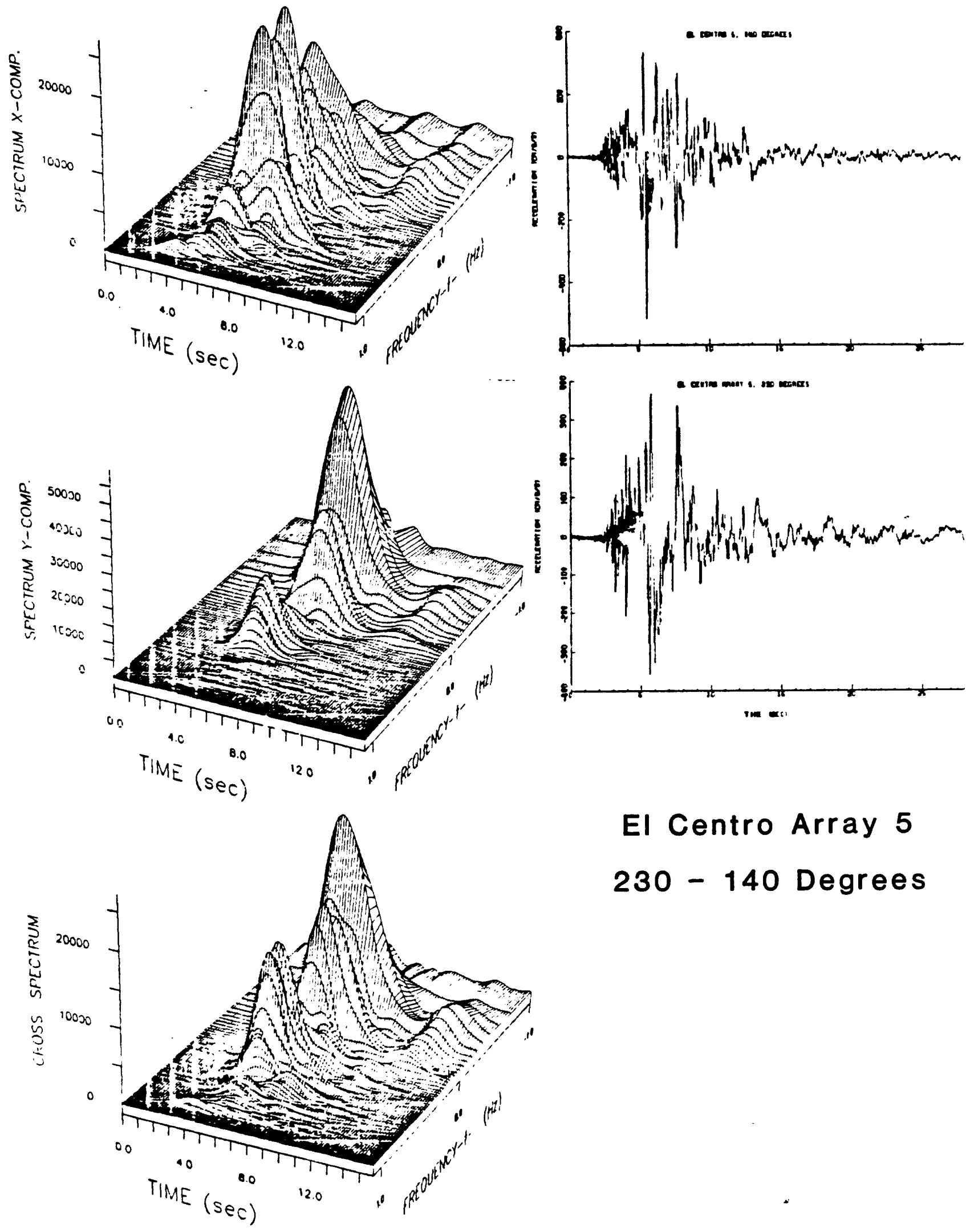

\section{El Centro Array 5 230 - 140 Degrees}

FIG. 2. (cont.) 

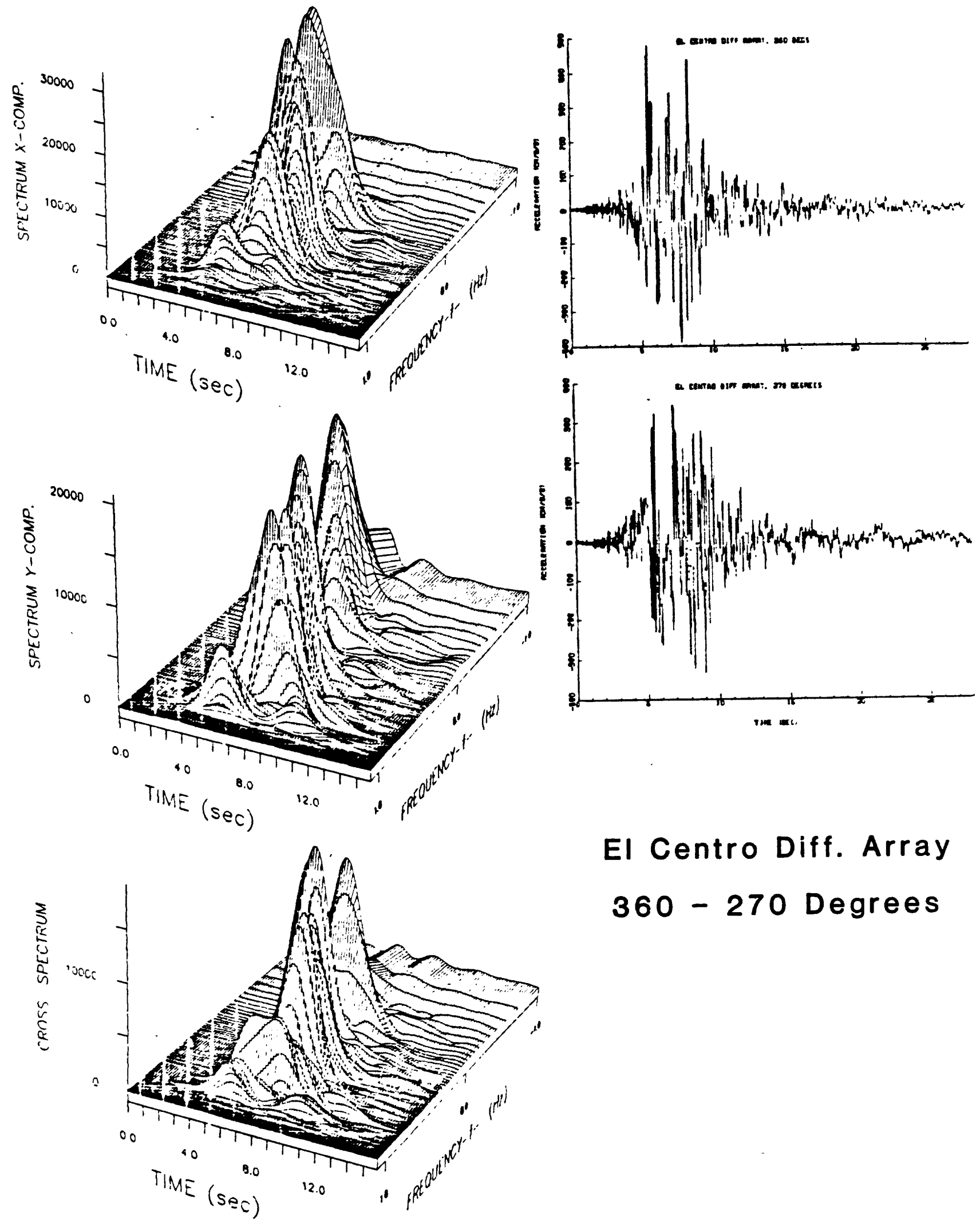

\section{El Centro Diff. Array 360 - 270 Degrees}

FIG. 2. (cont.) 

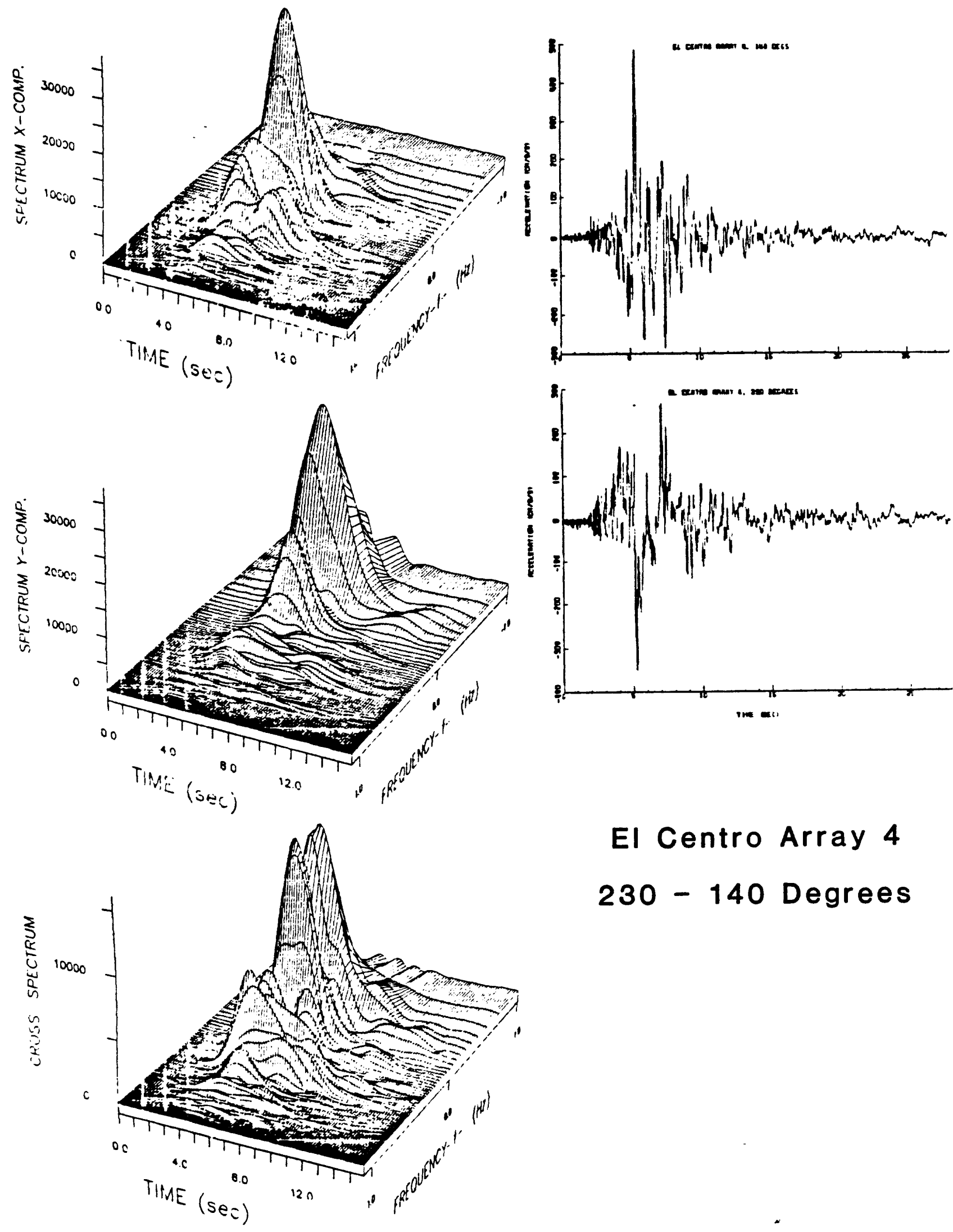

\section{El Centro Array 4 \\ 230 - 140 Degrees}

FIG. 2. (cont.) 

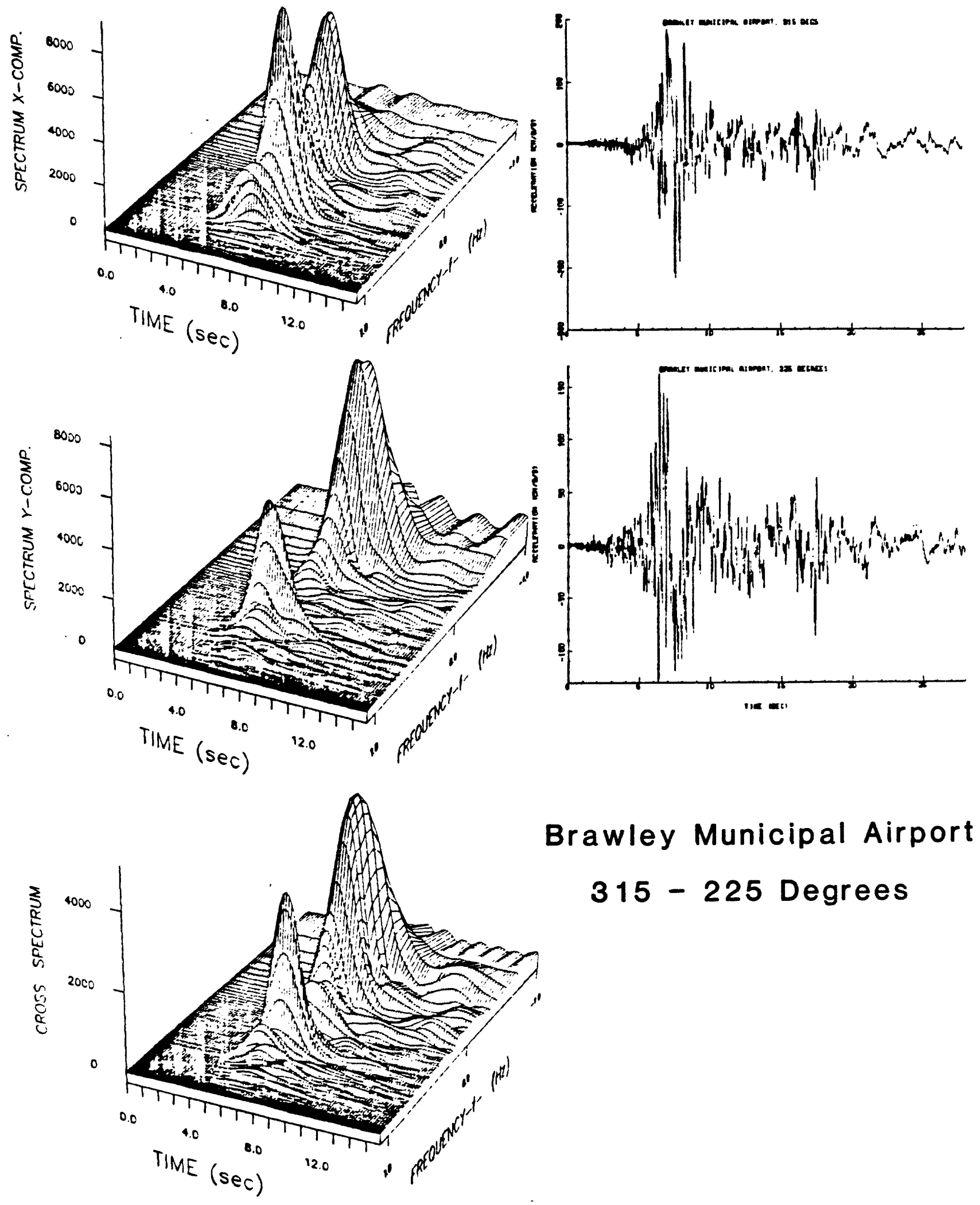

Brawley Municipal Airport 315 - 225 Degrees

FIG. 2. (cont.) 

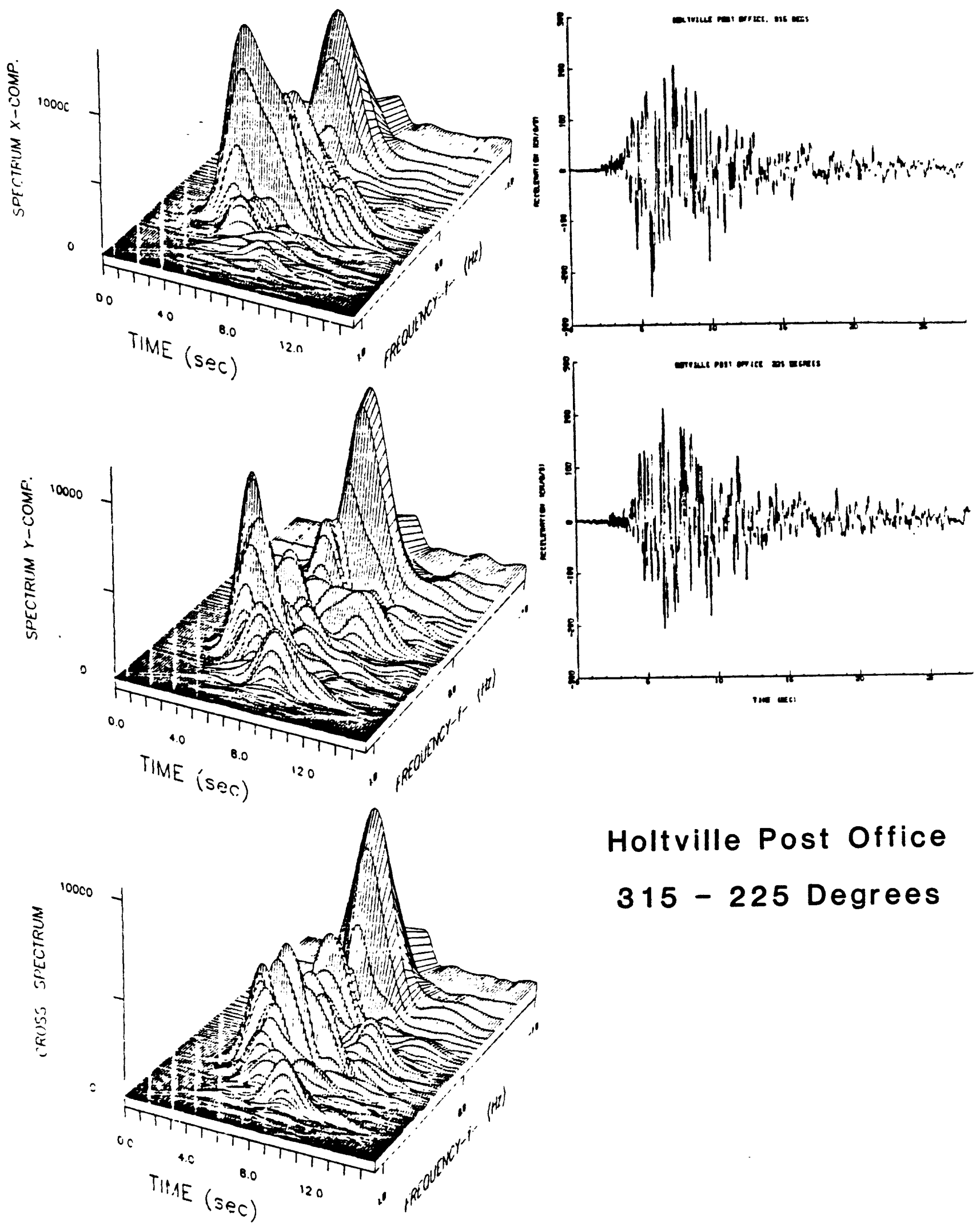

Holtville Post Office 315 - 225 Degrees

FIG. 2. (cont.) 

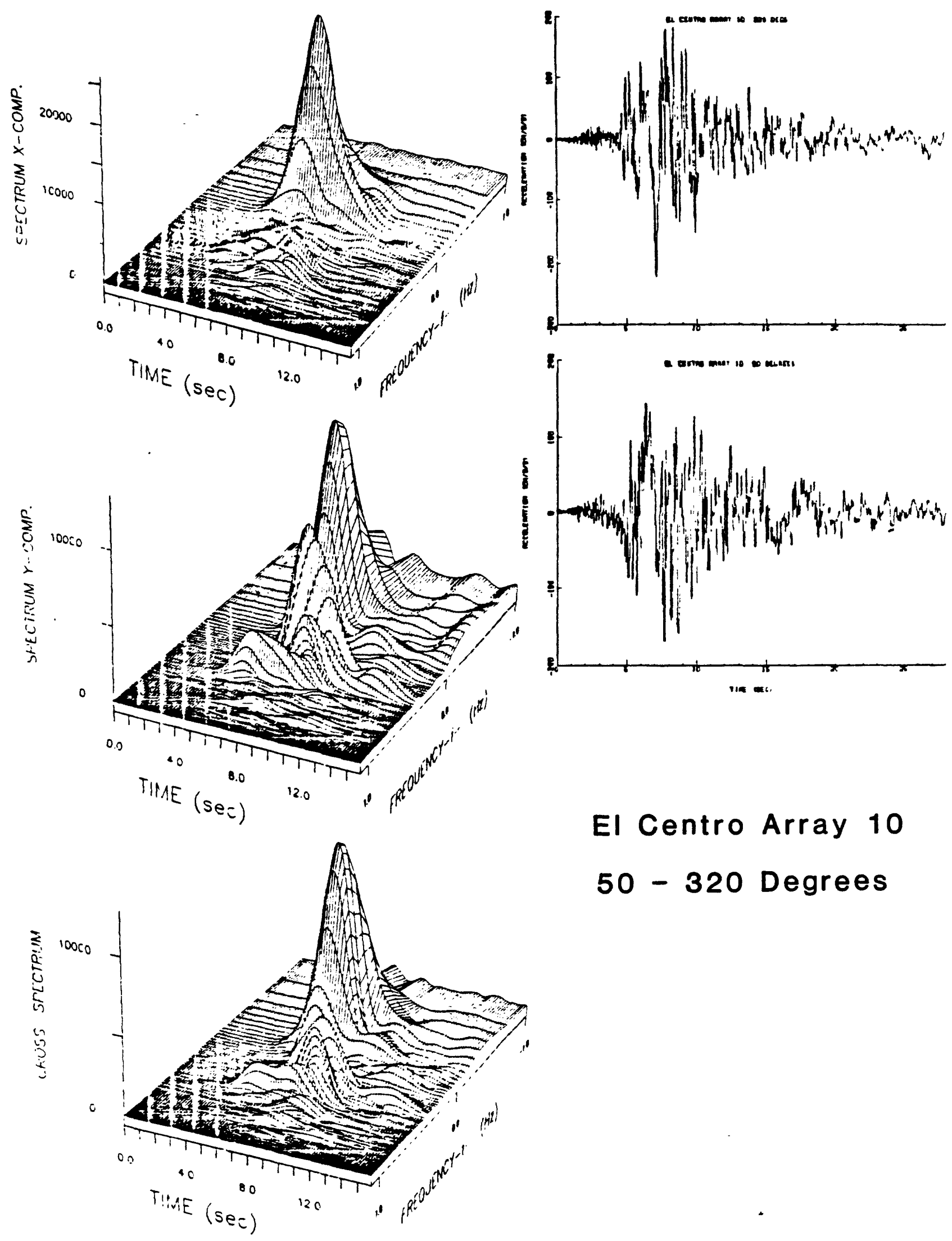

El Centro Array 10 50 - 320 Degrees

FIG. 2. (cont.) 

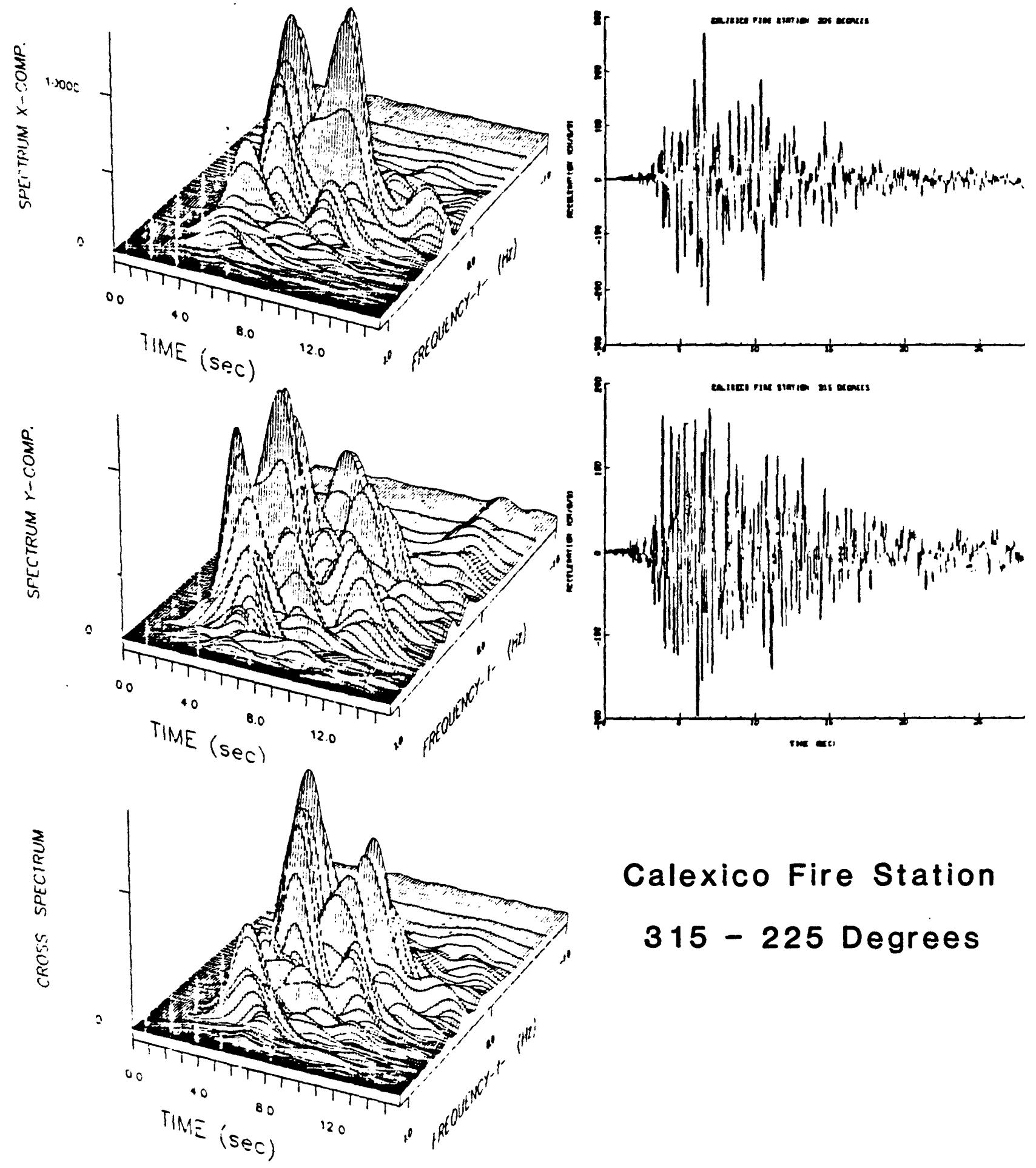

Calexico Fire Station

315 - 225 Degrees

FIG. 2. (cont.) 

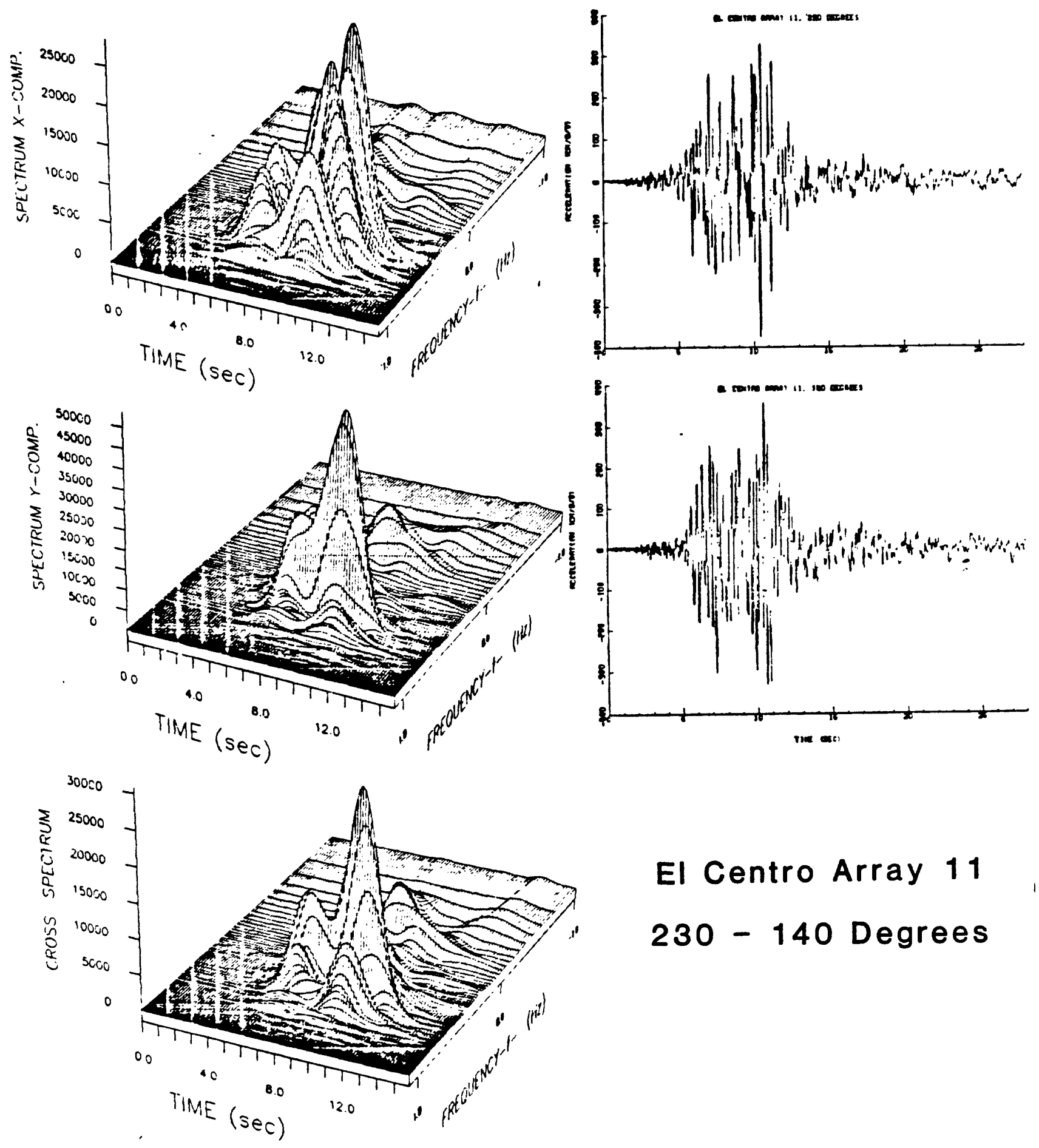

El Centro Array 11

230 - 140 Degrees

FIG. 2. (cont.) 

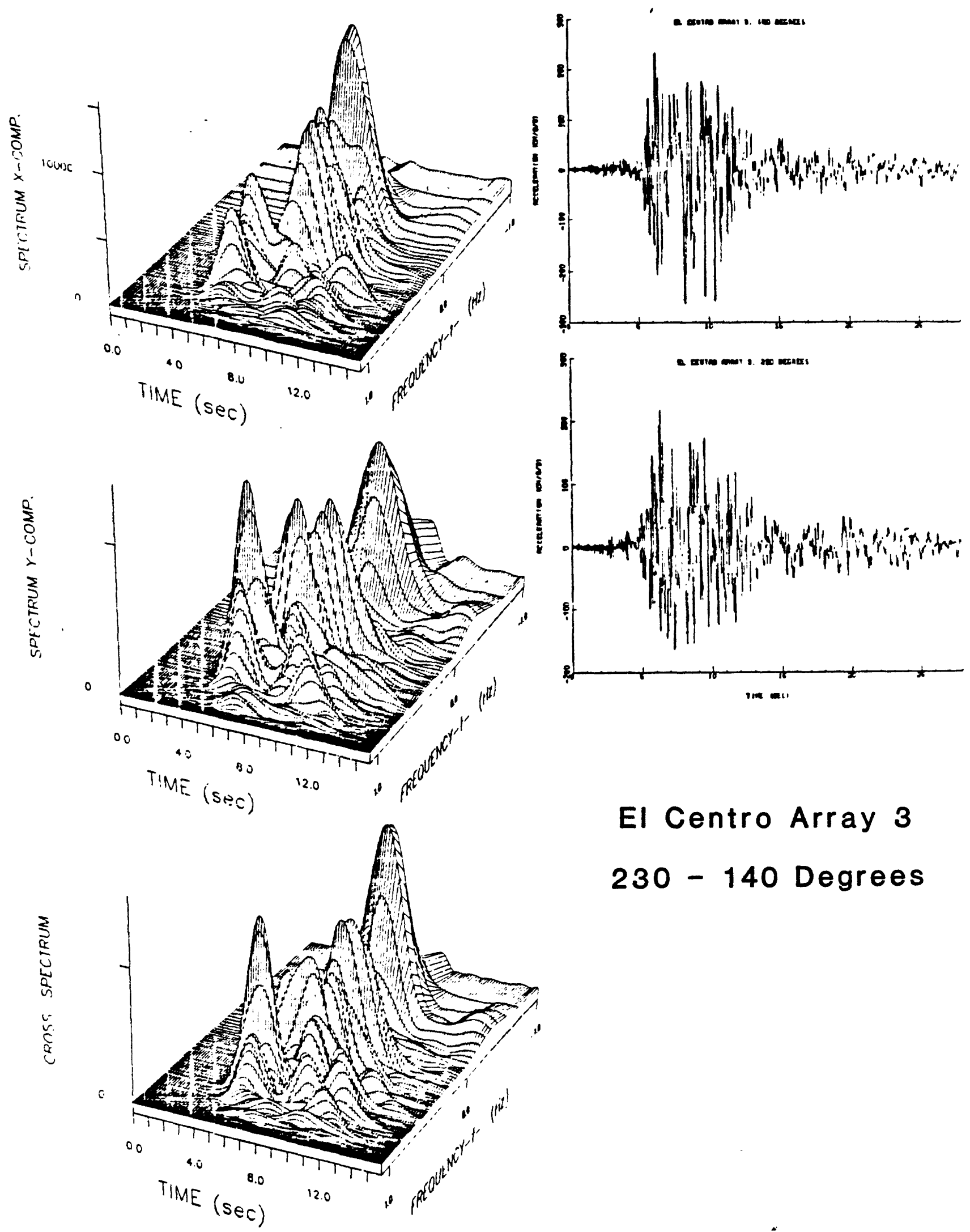

\section{El Centro Array 3 \\ 230 - 140 Degrees}

FIG. 2. (cont.) 

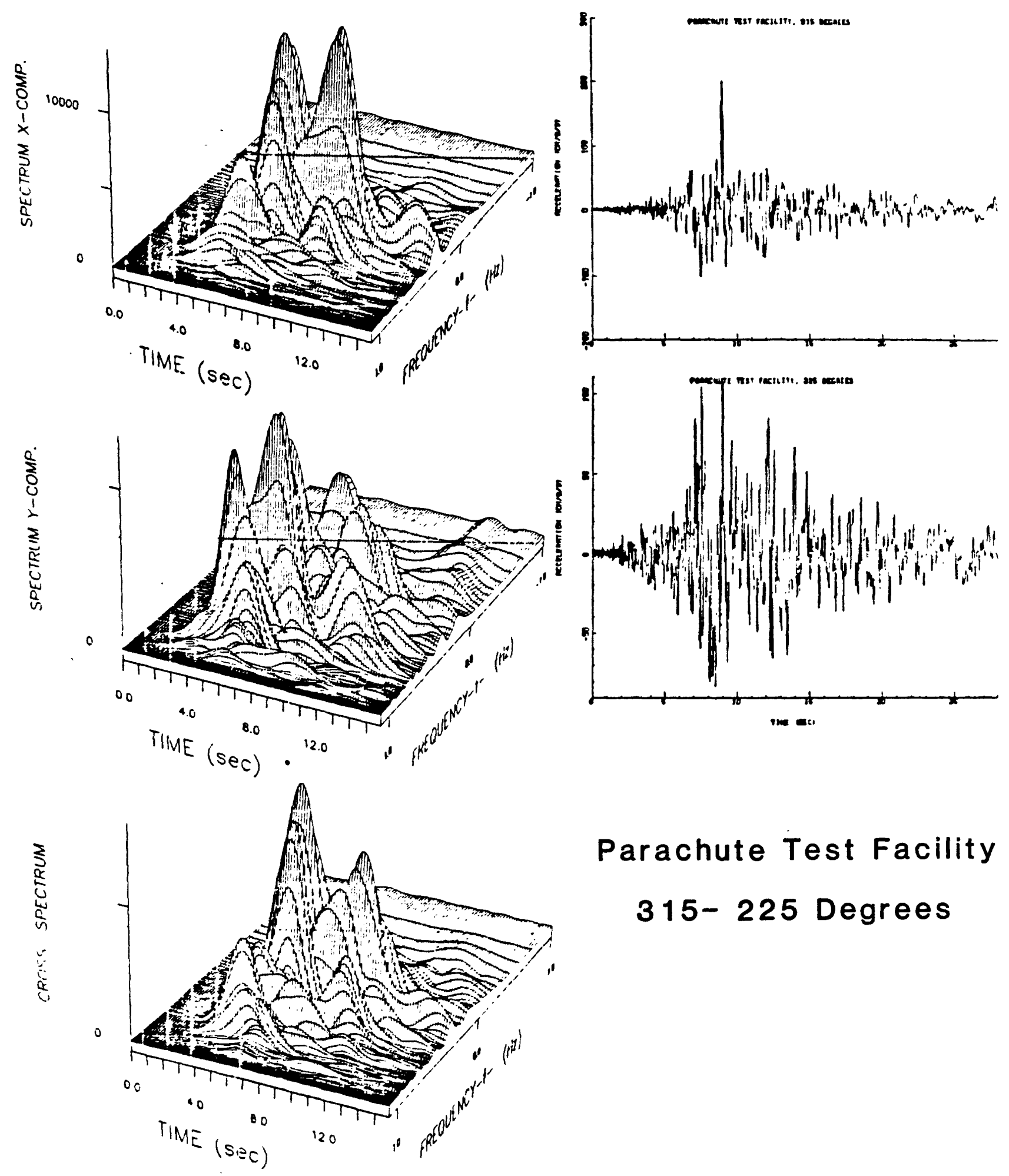

Parachute Test Facility

\section{5- 225 Degrees}

FIG. 2. (cont.) 

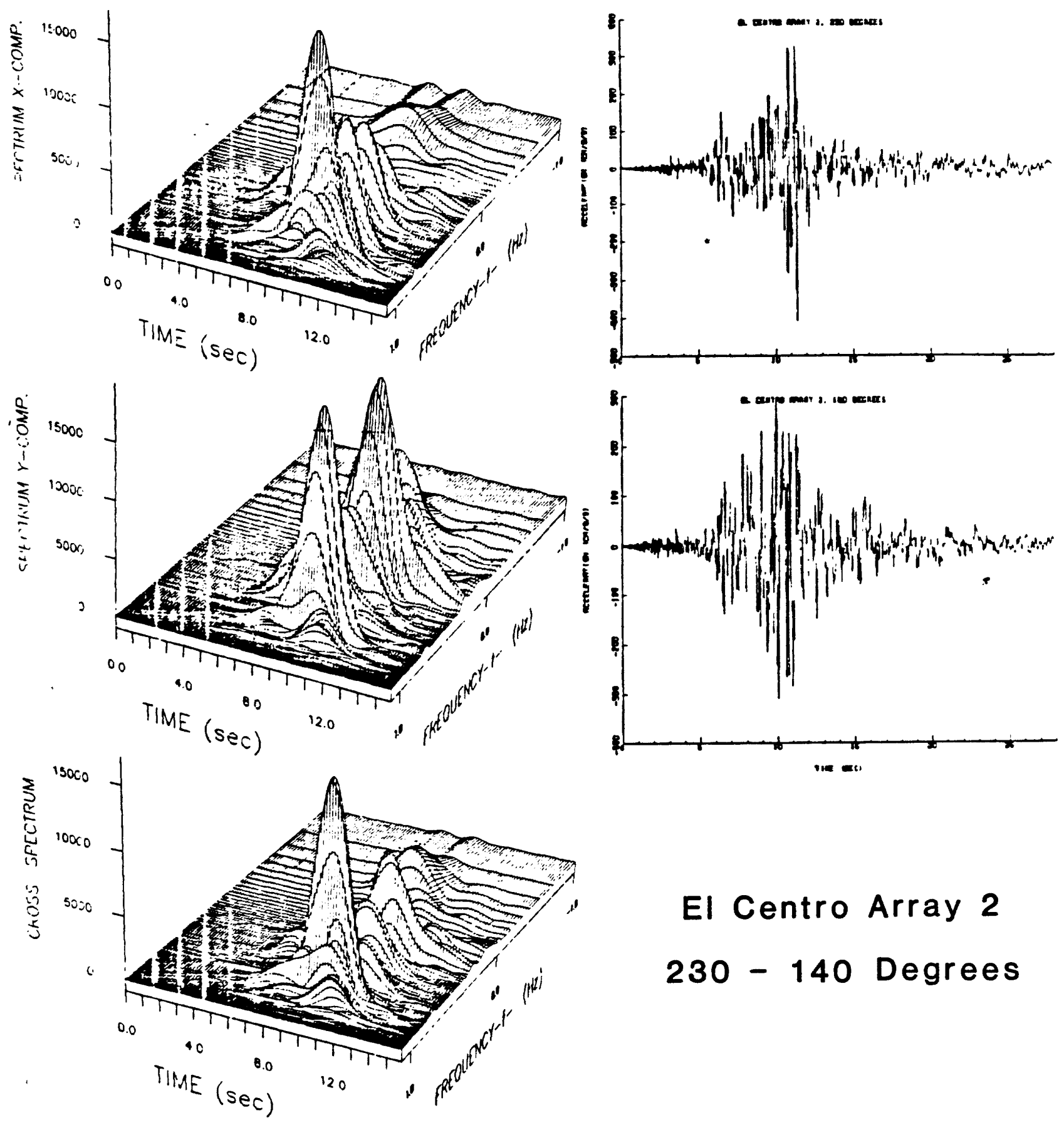

FIG. 2. (cont.) 

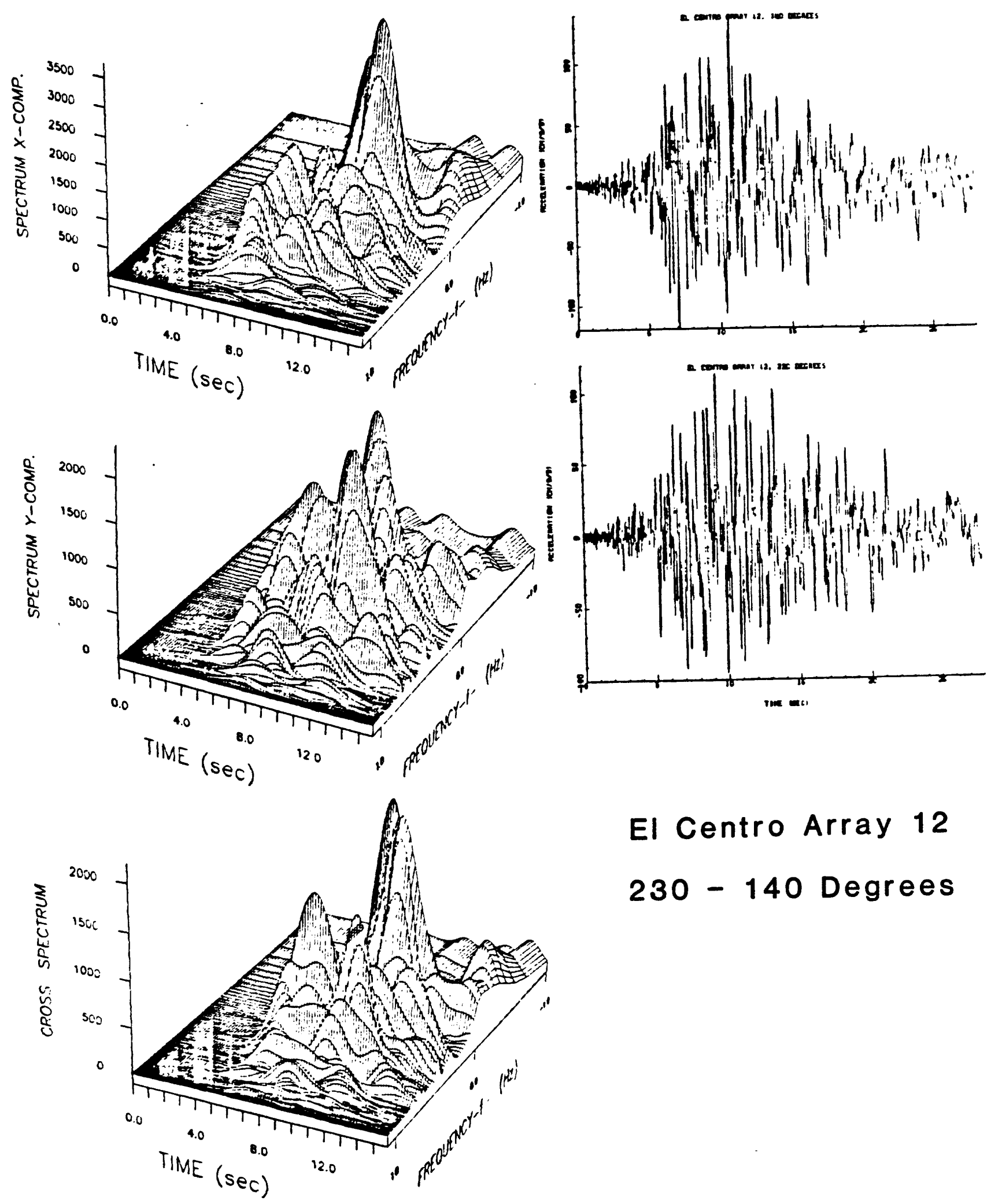

El Centro Array 12 230 - 140 Degrees

FIG. 2. (cont.). 

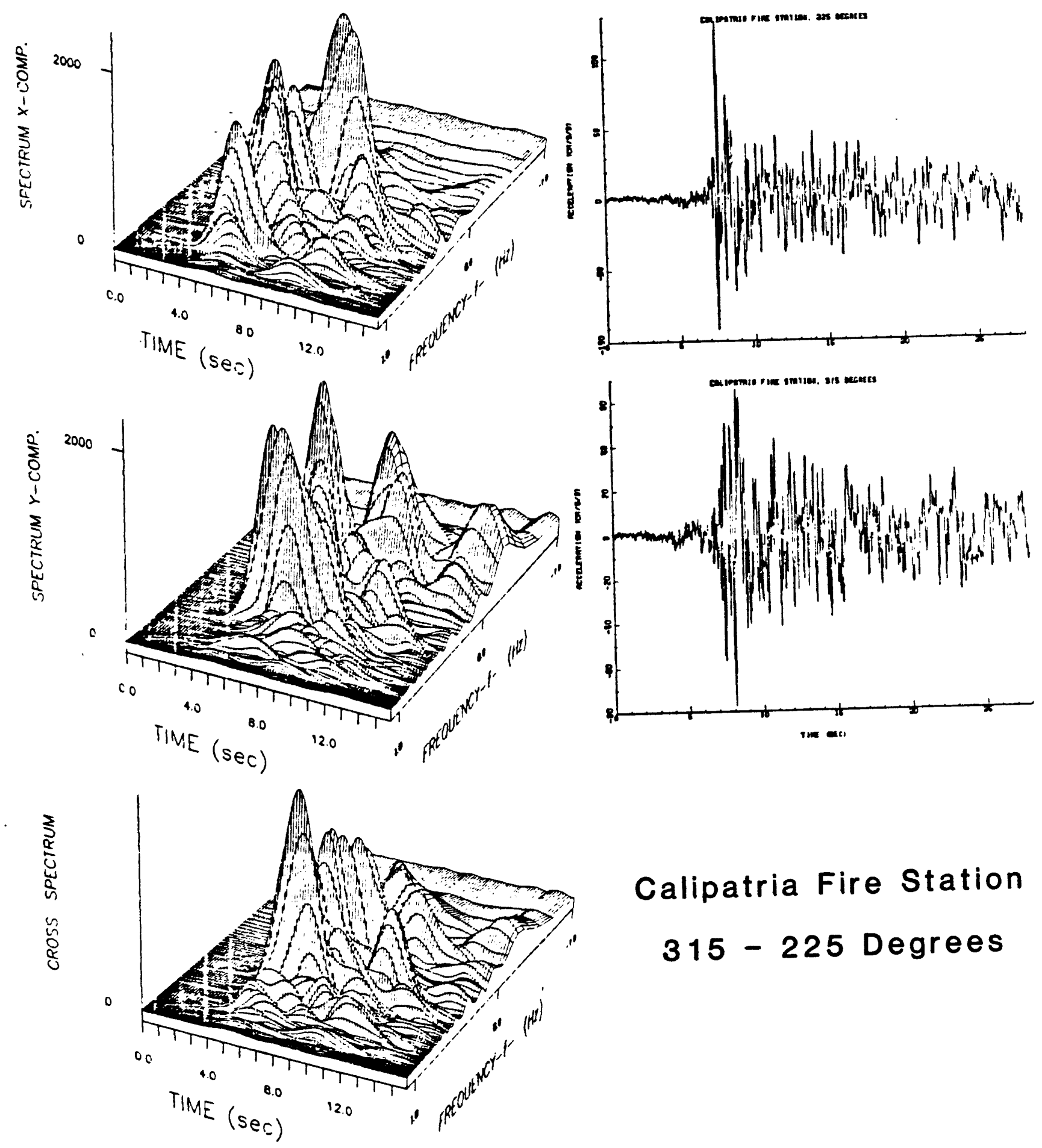

Calipatria Fire Station 315 - 225 Degrees

FIG. 2. (cont.) 

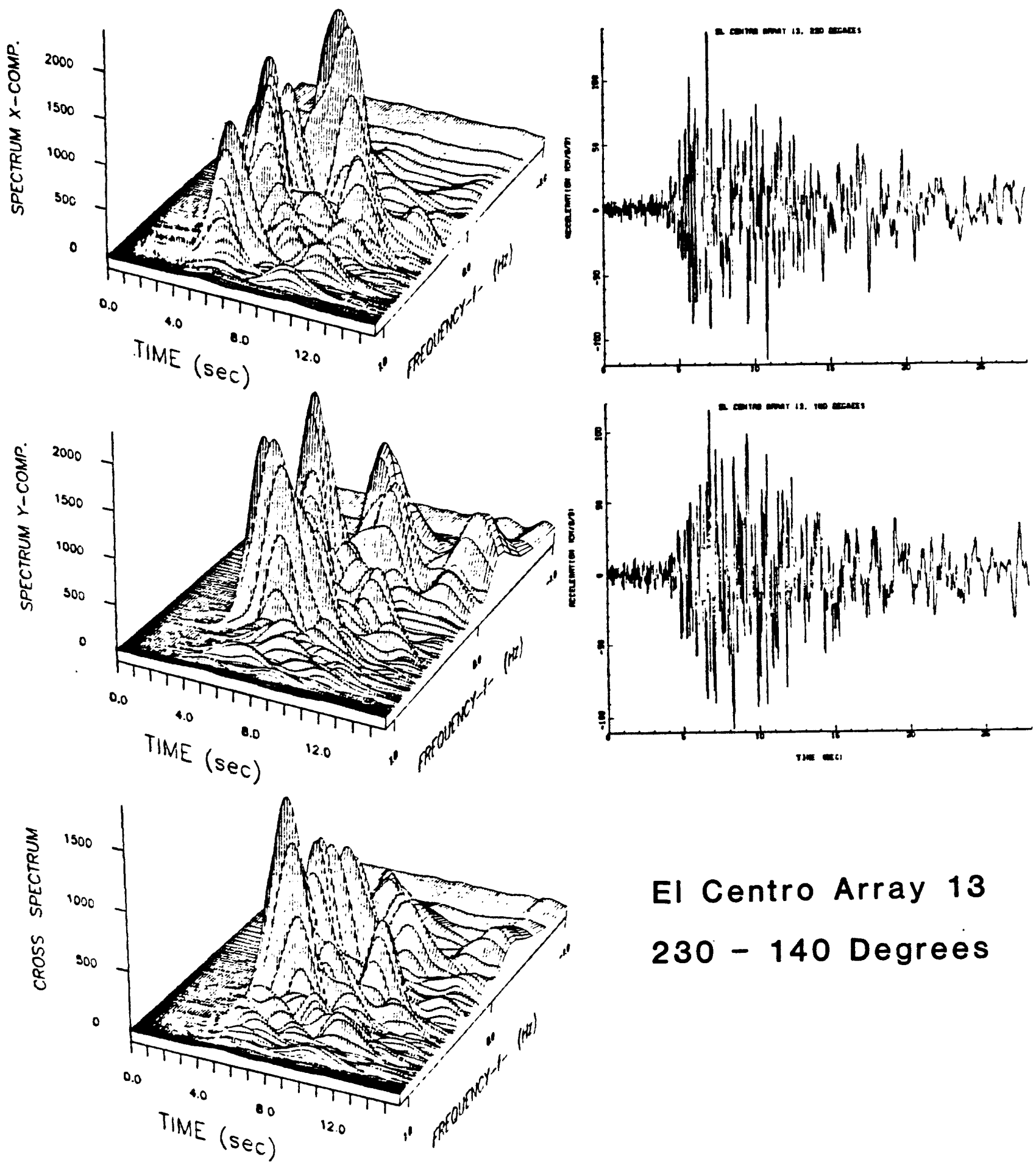

El Centro Array 13 230 - 140 Degrees

FIG. 2. (cont.) 

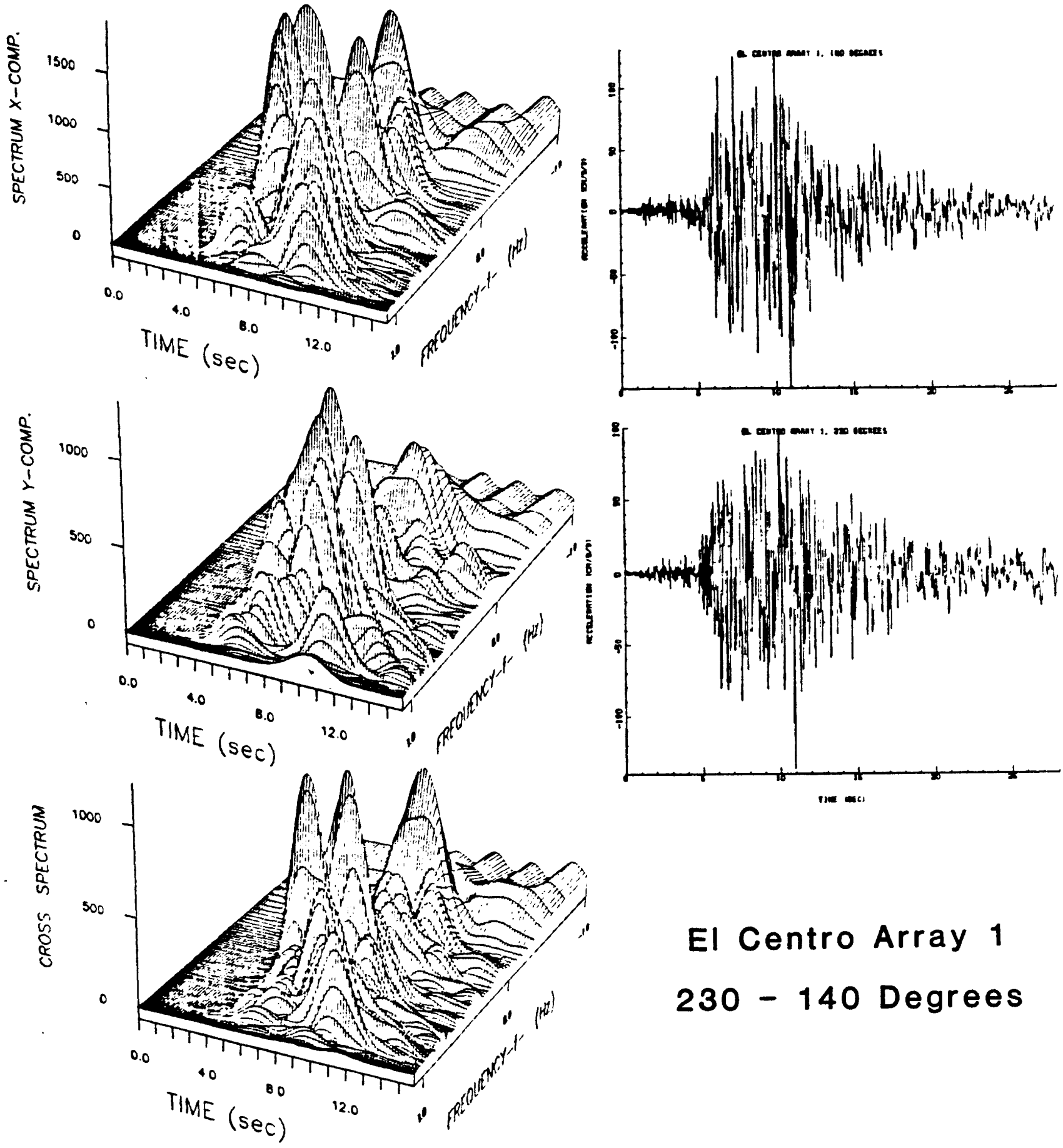

\section{El Centro Array 1 230 - 140 Degrees}

FIG. 2. (cont.) 

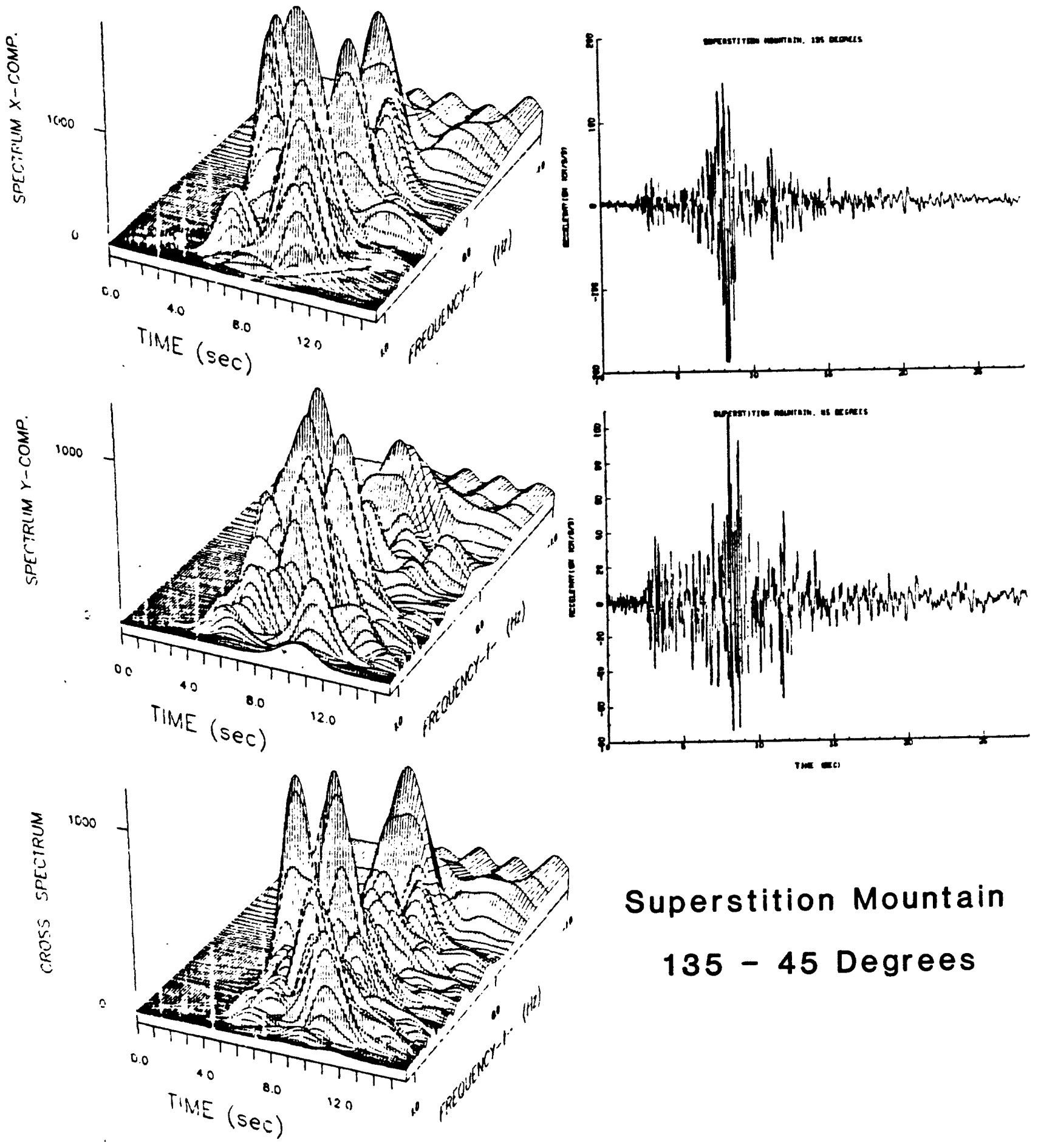

Superstition Mountain 135 - 45 Degrees

FIG. 2. (cont.) 

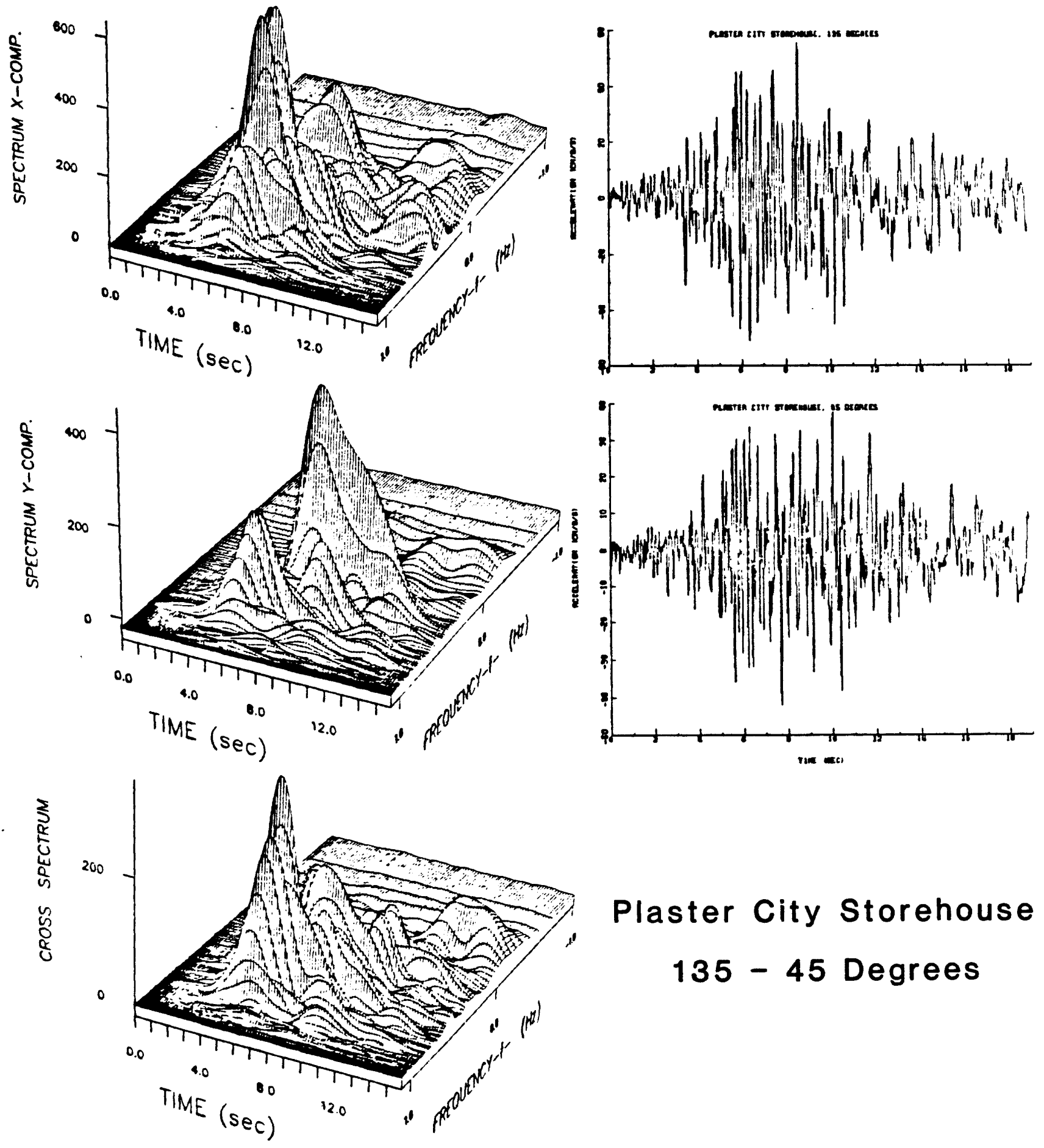

Plaster City Storehouse 135 - 45 Degrees

FIG. 2. (cont.) 

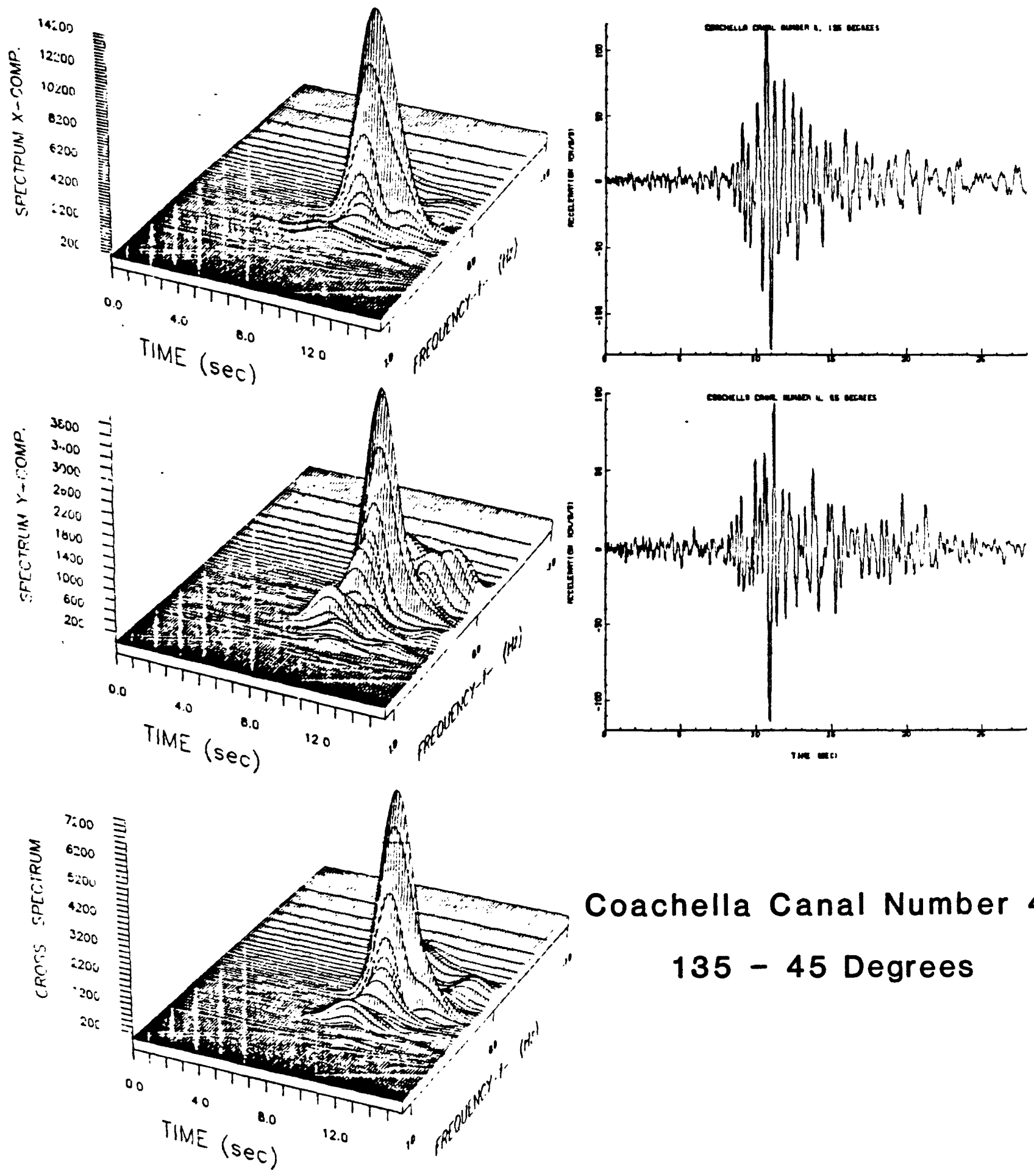

Coachella Canal Number 4 135 - 45 Degrees

FIG. 2. (cont.) 


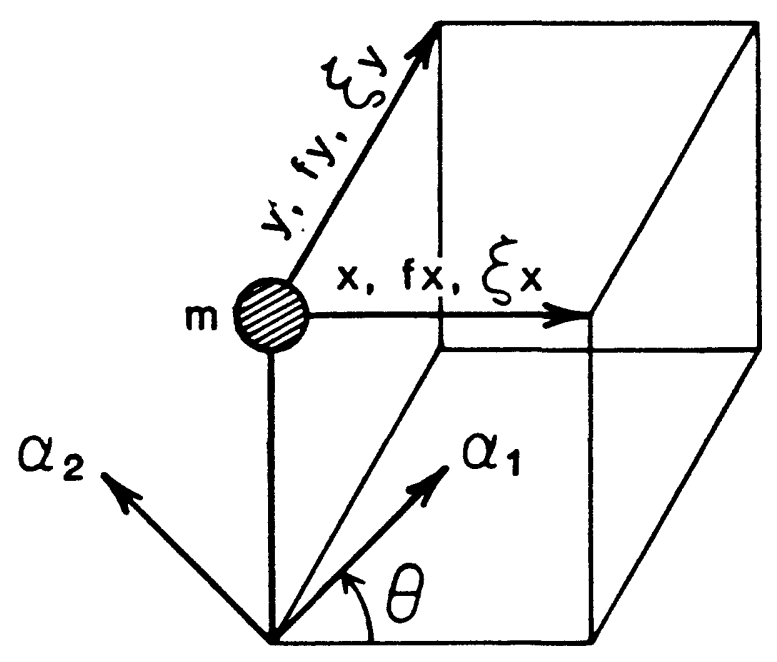

FIG. A.1. Schematic of 2-DOF oscillator subjected to arbitrarily oriented bi-directional base motion. 